\title{
QUANDLES ASSOCIATED TO GALOIS COVERS OF ARITHMETIC SCHEMES
}

\author{
Nobuyoshi TAKAHASHI \\ (Received 27 December 2017 and revised 23 May 2018)
}

\begin{abstract}
Let $X$ be a normal, separated and integral scheme of finite type over $\mathbb{Z}$ and $\mathcal{M}$ a set of closed points of $X$. To a Galois cover $\tilde{X}$ of $X$ unramified over $\mathcal{M}$, we associate a quandle whose underlying set consists of points of $\tilde{X}$ lying over $\mathcal{M}$. As the limit of such quandles over all étale Galois covers and all étale abelian covers, we define topological quandles $Q(X, \mathcal{M})$ and $Q^{\mathrm{ab}}(X, \mathcal{M})$, respectively. Then we study the problem of reconstruction. Let $K$ be $\mathbb{Q}$ or a quadratic field, $\mathcal{O}_{K}$ its ring of integers, $X=\operatorname{Spec} \mathcal{O}_{K} \backslash\{\mathfrak{p}\}$ the complement of a closed point such that $\pi_{1}(X)^{\mathrm{ab}}$ is infinite, and $\mathcal{M}$ a set of primes with Dirichlet density one. Using results from $p$-adic transcendental number theory, we show that $K, \mathfrak{p}$ and the projection $\mathcal{M} \rightarrow$ Spec $\mathbb{Z}$ can be recovered from the topological quandle $Q(X, \mathcal{M})$ or $Q^{\mathrm{ab}}(X, \mathcal{M})$.
\end{abstract}

\section{Introduction}

A quandle is a set $Q$ endowed with a binary operation $\triangleright$ which satisfies the following conditions:

(1) $q \triangleright q=q$;

(2) $s_{q}: Q \rightarrow Q ; r \mapsto q \triangleright r$ is a bijection;

(3) $q \triangleright(r \triangleright s)=(q \triangleright r) \triangleright(q \triangleright s)$.

While there were some previous works on similar algebraic structures, it began to be widely studied after the works of Joyce [J82] and Matveev [M82] relating them to knots. To any knot they associated a quandle, called the knot quandle. They used it to study invariants of the knot and proved that a knot is determined up to equivalence by its knot quandle.

If $K$ is a knot in $\mathbb{R}^{3}$, its knot quandle is the set of homotopy classes of 'nooses' or 'lollipops' with some fixed base point whose boundaries go around $K$ exactly once. If $[a]$ and $[b]$ are such classes and $\partial a$ denotes the boundary of $a$ regarded as a loop, then $[a] \triangleright[b]$ is defined as the class of the noose given by appending $\partial a$ to $b$. Roughly speaking, it is the class of $b$ transformed by the monodromy along $\partial a$.

In number theory, it has been noticed that there are various analogous phenomena between primes and knots (see e.g. [M12]). The purpose of this paper is to define a certain analogue of the knot quandle for arithmetic schemes and study how much information about an arithmetic scheme one can recover from the associated quandle. The latter can also be seen as a quandle analogue of anabelian geometry, i.e. the reconstruction of objects

2010 Mathematics Subject Classification: Primary 14G32; Secondary 20N02, 57M27.

Keywords: quandle; anabelian geometry.

(C) 2019 Faculty of Mathematics, Kyushu University 
in arithmetic geometry from their arithmetic fundamental groups (see [NSW08, Ch. XII], especially Question 12.3.4).

Let $X$ be an arithmetic scheme, which will mean a scheme of finite type over $\mathbb{Z}$ in this paper. We assume that it is normal, separated and integral. One important example is $\operatorname{Spec} \mathbb{Z} \backslash\{(p)\}$ where $p$ is a prime number. This can be seen as an analogue of the complement of a knot in $\mathbb{R}^{3}$. Let us fix a set $\mathcal{M}$ of closed points of $X$, which will be regarded as a collection of loops. Let $\tilde{X}$ be a Galois cover of $X$ unramified over $\mathcal{M}$. We allow 'Galois covers of infinite degree' to include in our consideration the maximal unramified cover $X^{\text {ur }}$ and the maximal unramified abelian cover $X^{\mathrm{ur}, \mathrm{ab}}$.

We define the set $Q(\tilde{X} / X, \mathcal{M})$ as the inverse image of $\mathcal{M}$ in $\tilde{X}$ and endow it with a quandle operation. In the analogy between primes and knots, Frobenius maps correspond to monodromies around loops. Thus for elements $x$ and $y$ of $Q(\tilde{X} / X, \mathcal{M})$, denote by $s_{x}$ the Frobenius automorphism of $\tilde{X}$ associated to $x$, and define $x \triangleright y$ to be $s_{x}(y)$. We also define a topology on $Q(\tilde{X} / X, \mathcal{M})$ and show that $Q(\tilde{X} / X, \mathcal{M})$ is a topological quandle.

By choosing a canonically defined Galois cover such as $X^{\mathrm{ur}}$ or $X^{\mathrm{ur}, \mathrm{ab}}, Q(\tilde{X} / X, \mathcal{M})$ can be considered as an invariant of the pair $(X, \mathcal{M})$. The quandle $Q\left(X^{\mathrm{ur}} / X, \mathcal{M}\right)$ is not an exact analogue of a knot quandle, but is related to certain quandles defined from loops in knot complements. See Remarks 3.7 and 4.17 for how our construction and results are related to those in topology.

Let $\tilde{X}$ be either $X^{\text {ur }}$ or $X^{\text {ur,ab }}$. We study how much information about $X$ and $\mathcal{M}$ one can recover from $Q=Q(\tilde{X} / X, \mathcal{M})$. Write $G$ for the group $\operatorname{Aut}(\tilde{X} / X)$. Note that the quandle $Q$ has some explicit information about points of $\tilde{X}$, which $G$ does not. On the other hand, it is not obvious when $G$ can be reconstructed from $Q$.

We restrict to the case of an arithmetic curve, i.e. an arithmetic scheme $X=\operatorname{Spec} \mathcal{O}_{K} \backslash S$ where $K$ is a number field and $S$ is a finite set of primes. It is easier to recover the information when we consider sufficiently many loops. We assume that $\mathcal{M}$ is of density one, where 'density' in this paper shall mean the Dirichlet density in the set of all primes in $K$. Then the Galois group $G$, and hence $\mathcal{M}=Q / G$ as a set, can be reconstructed from the topological quandle $Q$ (Corollary 4.3). In particular, $Q\left(X^{\mathrm{ur}, \mathrm{ab}} / X, \mathcal{M}\right)$ has a description in terms of $Q\left(X^{\mathrm{ur}} / X, \mathcal{M}\right)$.

We further specialize to the case where $K$ is rational or quadratic, $S$ consists of one prime and $\pi_{1}^{\mathrm{ab}}(X)$ is infinite. Then our main theorem shows that pretty much can be recovered from $Q\left(X^{\mathrm{ur}, \mathrm{ab}} / X, \mathcal{M}\right)$.

THEOREM 1.1. (Theorem 4.11) Let each of $K$ and $K^{\prime}$ be either $\mathbb{Q}$ or a quadratic field, $\mathfrak{p}$ and $\mathfrak{p}^{\prime}$ primes in $K$ and $K^{\prime}$ respectively, and $\mathcal{M}$ and $\mathcal{M}^{\prime}$ sets of primes with density one in $K$ and $K^{\prime}$, respectively.

Write $X=\operatorname{Spec} \mathcal{O}_{K} \backslash\{\mathfrak{p}\}$ and $Q=Q\left(X^{\mathrm{ur}, \mathrm{ab}} / X, \mathcal{M}\right)$. Define $X^{\prime}$ and $Q^{\prime}$ in the same way. Assume that $\pi_{1}^{\mathrm{ab}}(X)$ or $\pi_{1}^{\mathrm{ab}}\left(X^{\prime}\right)$ is infinite.

If $\varphi: Q \rightarrow Q^{\prime}$ is an isomorphism of topological quandles, then there exists an isomorphism $\sigma: K \rightarrow K^{\prime}$ which maps $\mathfrak{p}$ to $\mathfrak{p}^{\prime}$, and $\varphi$ commutes with the natural maps to $\operatorname{Spec} \mathbb{Z}$.

Furthermore, except in the real quadratic case, $\sigma$ can be chosen so that $\varphi$ is compatible with the isomorphism $X \rightarrow X^{\prime}$ induced by $\sigma$. 
The basic idea for our proof is as follows. The Galois group $\pi_{1}^{\mathrm{ab}}(X)$ is already recovered in Corollary 4.3, and the quandle structure gives us a map $r: \mathcal{M} \rightarrow \pi_{1}^{\mathrm{ab}}(X)$, which is essentially the global reciprocity map in class field theory. According to class field theory, there is a natural homomorphism $\mathcal{O}_{K_{\mathfrak{p}}}^{\times} \rightarrow \pi_{1}^{\mathrm{ab}}(X)$ compatible with $r$, with finite cokernel. If available, this could be used as a ruler to help in recovering $\mathfrak{l}$ from $r(\mathfrak{l})$. Unfortunately, our reconstruction of $\pi_{1}^{\mathrm{ab}}(X)$ from $Q$ does not directly provide us with this map. Instead we may think of $r(\mathcal{M})$ as a sort of unlabelled scale: we look at 'arrangements' $\left\{r\left(\mathfrak{l}_{1}\right), \ldots, r\left(\mathfrak{l}_{k}\right)\right\} \subset$ $\pi_{1}^{\mathrm{ab}}(X)$ for $\mathfrak{l}_{1}, \ldots, \mathfrak{l}_{k} \in \mathcal{M}$. Applying results from $p$-adic transcendental number theory, the $p$-adic six-exponentials theorem and its generalizations, to certain ratios formed from $r\left(\mathfrak{l}_{i}\right)$, we show that if an isomorphism $\pi_{1}^{\mathrm{ab}}(X) \rightarrow \pi_{1}^{\mathrm{ab}}\left(X^{\prime}\right)$ is compatible with a bijection $\mathcal{M} \rightarrow \mathcal{M}^{\prime}$, then the conclusion of the theorem must hold.

Remark 1.2. Let us compare our approaches and results with those of previous works.

(1) In Tamagawa's reconstruction [T97] of affine curves over finite fields from their Galois groups, the decomposition groups of points are characterized by group-theoretical properties, and the function field is recovered from these groups. In our case, while the map $r$ gives us decomposition groups at many unramified primes together with natural generators, we do without recovering the decomposition groups at ramified points. All we do here is to prove that there is no unexpected isomorphism of associated quandles, with the help of transcendental number theory. As a drawback, we do not know how to directly reconstruct $K$ as a set at this moment.

(2) Unlike in the case of curves over fields, there seem to be few works on reconstruction of arithmetic curves. Ivanov [I14] studies the problem of reconstruction of an arithmetic curve from its arithmetic fundamental group under the assumption that at least two rational primes are invertible in $\mathcal{O}_{K, S}$ (or, equivalently, lie completely below $S$ ).

(3) Mochizuki [M08, Theorem 6.4] associates a certain category $\mathcal{C}$, called an arithmetic Frobenioid, to a number field $F$ and its Galois extension $\tilde{F}$, and reconstructs $F$ and $\tilde{F}$ from the associated arithmetic Frobenioid $\mathcal{C}$.

We note an intriguing similarity in the proofs of this theorem and ours, although the author does not know exactly how they are related. The proof in [M08, Theorem 6.4] applies the archimedean (i.e. original) six-exponentials theorem in order to recover residue characteristics of points, while we use the $p$-adic six-exponentials theorem.

The rest of this paper is organized as follows. In Section 2, we give a brief introduction to quandles and topological quandles. In Section 3, we associate a topological quandle to a Galois cover of an arithmetic scheme and a set of unbranched points. Then we restrict to the case of arithmetic curves and prove results on reconstruction in Section 4. Finally, we study the automorphism groups of quandles associated to Galois covers in Section 5.

\section{Quandles}

Let us recall the definition of a quandle. Note that our convention is a little different from that of Joyce [J82].

Definition 2.1. A quandle is a set $Q$ equipped with a binary operation $\triangleright$ satisfying the following axioms.

(1) For any $q \in Q, q \triangleright q=q$ holds. 
(2) For any $q, r \in Q$, there exists a unique element $r^{\prime} \in Q$ such that $q \triangleright r^{\prime}=r$.

(3) For any $q, r, s \in Q, q \triangleright(r \triangleright s)=(q \triangleright r) \triangleright(q \triangleright s)$.

We define $s_{q}: Q \rightarrow Q$ by $s_{q}(r)=q \triangleright r$, which is bijective by axiom (2), and write $q \triangleright^{-1} r$ for $s_{q}^{-1}(r)$.

Definition 2.2. We define the automorphism group of $Q$ as

$$
\operatorname{Aut}(Q):=\{f: Q \rightarrow Q \mid f(q \triangleright r)=f(q) \triangleright f(r) \text { and } f \text { is bijective }\},
$$

the inner automorphism group of $Q$ as

$$
\operatorname{Inn}(Q):=\left\langle s_{q} \mid q \in Q\right\rangle
$$

and the group of transvections as

$$
\operatorname{Tr}(Q):=\left\{s_{q_{1}}^{e_{1}} \cdots s_{q_{n}}^{e_{n}} \mid n \in \mathbb{N}, q_{1}, \ldots, q_{n} \in Q, \sum_{i=1}^{n} e_{i}=0\right\} .
$$

Since $s_{q}$ is an automorphism of $Q$ by axioms (2) and (3), $\operatorname{Inn}(Q)$ is in fact a subgroup of $\operatorname{Aut}(Q)$.

Example 2.3. If $G$ is a group, each of the operations $g \triangleright h:=g^{-1} h g$ and $g \triangleright h:=g h g^{-1}$ makes $G$ a quandle. Such a quandle is called a conjugation quandle.

Example 2.4. Let $G$ be a group, $\left\{z_{\lambda}\right\}_{\lambda \in \Lambda}$ a family of elements of $G$ parametrized by $\Lambda$ and $\left\{H_{\lambda}\right\}_{\lambda \in \Lambda}$ a family of subgroups of $G$ such that $z_{\lambda} \in H_{\lambda}$ and $H_{\lambda}$ is contained in the centralizer of $z_{\lambda}$.

On the set $Q:=\bigsqcup_{\lambda \in \Lambda} G / H_{\lambda}$, define the following binary operation. If $x$ and $y$ are elements of $G$, and $\lambda$ and $\mu$ are elements of $\Lambda$, then

$$
x H_{\lambda} \triangleright y H_{\mu}:=x z_{\lambda} x^{-1} y H_{\mu} .
$$

This operation makes $Q$ a quandle, which we denote by $Q\left(G,\left\{H_{\lambda}\right\},\left\{z_{\lambda}\right\}\right)$.

Definition 2.5. An augmented quandle is a pair $(Q, G)$ of a set $Q$ and a group $G$ together with an action of $G$ on $Q$ and a map $\varepsilon: Q \rightarrow G$ (called the augmentation map) satisfying the following conditions:

- $\varepsilon(q) q=q$;

- $\varepsilon(g q)=g \varepsilon(q) g^{-1}$.

Then we define an operation $\triangleright$ by $q \triangleright r:=\varepsilon(q) r$, which is easily seen to satisfy the quandle axioms.

If $(Q, G)$ and $\left(Q^{\prime}, G^{\prime}\right)$ are augmented quandles, a homomorphism of augmented quandles from $(Q, G)$ to $\left(Q^{\prime}, G^{\prime}\right)$ is a pair of maps $Q \rightarrow Q^{\prime}$ and $G \rightarrow G^{\prime}$ compatible with the actions and the augmentation maps.

Remark 2.6. (1) It also follows that $G$ acts on $Q$ by quandle automorphisms and $\varepsilon$ is a quandle homomorphism if we regard $G$ as a conjugation quandle.

(2) It is easy to see that $(Q, \operatorname{Aut}(Q))$ and $(Q, \operatorname{Inn}(Q))$ are augmented quandles by the mapping $q \mapsto s_{q}$ and that the associated operations coincide with the original operation on $Q$.

Next we introduce notions of topological quandle and topological augmented quandle. 
Definition 2.7. A topological quandle is a topological space $Q$ endowed with a quandle operation $\triangleright$ such that $Q \times Q \rightarrow Q ;(q, r) \mapsto q \triangleright r$ and $Q \times Q \rightarrow Q ;(q, r) \mapsto q \triangleright^{-1} r$ are continuous.

A topological augmented quandle is a pair $(Q, G)$ of a topological space $Q$ and a topological group $G$ together with a continuous action of $G$ on $Q$ and a continuous map $\varepsilon: Q \rightarrow G$ satisfying $\varepsilon(q) q=q$ and $\varepsilon(g q)=g \varepsilon(q) g^{-1}$. Again, $Q$ is a topological quandle by the operation $q \triangleright r:=\varepsilon(q) r$.

Remark 2.8. In the definition of topological quandles in [R07, Definition 2.1], it is only assumed that $(q, r) \mapsto q \triangleright r$ is continuous and $s_{q}$ is a homeomorphism for any $q \in Q$. The author does not know if this definition is equivalent to ours.

Proposition 2.9. Let $(Q, G)$ be an augmented quandle and let $\left\{q_{\lambda}\right\}_{\lambda \in G \backslash Q}$ be a complete system of representatives for the quotient set $G \backslash Q$. Then there is a natural isomorphism of $Q\left(G,\left\{\operatorname{Stab}_{G}\left(q_{\lambda}\right)\right\},\left\{\varepsilon\left(q_{\lambda}\right)\right\}\right)$ and $Q$.

If $(Q, G)$ is a topological augmented quandle, $Q$ is Hausdorff, each $G$-orbit in $Q$ is open and $G$ is compact, then the above isomorphism is also an isomorphism of topological quandles.

Proof. For discrete quandles, the proof is the same as that of [J82, Theorem 7.2].

In the continuous case, let $\pi: G \rightarrow G / \operatorname{Stab}_{G}\left(q_{\lambda}\right)$ be the natural map, define $a: G \rightarrow G q_{\lambda}$ by $a(g)=g q_{\lambda}$ and write $\bar{a}: G / \operatorname{Stab}_{G}\left(q_{\lambda}\right) \rightarrow G q_{\lambda}$ for the induced bijection. The continuity of $\bar{a}$ follows from that of $a$. If $K$ is a closed subset of $G / \operatorname{Stab}_{G}\left(q_{\lambda}\right)$, then $\pi^{-1}(K)$ is compact. Since $Q$ is Hausdorff, $\bar{a}(K)=a\left(\pi^{-1}(K)\right)$ is closed. Thus $\bar{a}$ is a homeomorphism.

Since $G q_{\lambda}$ is open in $Q$, the natural map $Q\left(G,\left\{\operatorname{Stab}_{G}\left(q_{\lambda}\right)\right\},\left\{\varepsilon\left(q_{\lambda}\right)\right\}\right) \rightarrow Q$ is a homeomorphism.

\section{Quandles associated to Galois covers}

Let $X$ be a normal, separated and integral scheme of finite type over $\mathbb{Z}$. Let $K=K(X)$ be the function field of $X$ and $L$ an algebraic extension of $K$, possibly of infinite degree.

We denote by $X_{L}$ the normalization of $X$ in $L$. Explicitly, it can be described as follows. For an open affine subset Spec $A$ of $X$, let $A_{L}$ be the integral closure of $A$ in $L$. If $f$ is an element of $A$, the integral closure $\left(A_{f}\right)_{L}$ of $A_{f}$ in $L$ is equal to $\left(A_{L}\right)_{f}$, the localization of $A_{L}$ by $f$. This allows one to glue the affine schemes Spec $A_{L}$ together into a scheme $X_{L}$.

Let $X_{L}^{0}$ denote the set of closed points of $X_{L}$. This set can be described in terms of finite extensions. Let

$$
\mathcal{F}(L / K):=\{M \mid M \text { is an intermediate field of } L / K \text { which is finite over } K\}
$$

and

$$
\mathcal{G}(L / K):=\{M \in \mathcal{F}(L / K) \mid M \text { is a Galois extension of } K\} .
$$

For a point $P$ of a scheme, let $\mathbb{F}(P)$ denote the residue field at $P$.

\section{PROPOSITION 3.1.}

(1) If $L \supseteq M \supseteq K$ are algebraic field extensions, the natural map $\varphi_{L, M}: X_{L} \rightarrow X_{M}$ is surjective, and $\varphi_{L, M}^{-1}\left(X_{M}^{0}\right)=X_{L}^{0}$. 
(2) A point $x \in X_{L}$ is closed if and only if $\mathbb{F}(x)$ is algebraic over a finite field.

(3) There is a natural bijection between $X_{L}^{0}$ and $\lim _{M \in \mathcal{F}(L / K)} X_{M}^{0}$. If $L$ is a Galois extension of $K$, then $X_{L}^{0}$ is also in bijection with $\lim _{M \in \mathcal{G}(L / K)} X_{M}^{0}$.

Proof. (1) The surjectivity follows from the local description of $X_{L}$ above and the lying-over theorem. A point $x$ of $X_{L}$ is closed if and only if it is closed in any affine open neighborhood of the form Spec $A_{L}$. The latter condition is equivalent to saying that $\varphi_{L, K}(x)$ is closed in Spec $A$ by the lying-over and going-up theorem. Thus $x$ is closed in $X_{L}$ if and only if $\varphi_{L, K}(x)$ is closed in $X$, i.e. $\varphi_{L, K}^{-1}\left(X_{K}^{0}\right)=X_{L}^{0}$. Hence $\varphi_{L, M}^{-1}\left(X_{M}^{0}\right)=X_{L}^{0}$ holds.

(2) It is well known that a point $P$ of a scheme of finite type over $\mathbb{Z}$ is closed if and only if $\mathbb{F}(P)$ is finite. Since $\mathbb{F}(P)$ is the quotient field of a finitely generated $\mathbb{Z}$-algebra, it is finite if and only if it is algebraic over a finite field.

Let $x$ be a point of $X_{L}$. Then $\mathbb{F}(x)$ is algebraic over $\mathbb{F}\left(\varphi_{L, K}(x)\right)$. If $x$ is closed, then $\varphi_{L, K}(x)$ is closed by (1) and $\mathbb{F}\left(\varphi_{L, K}(x)\right)$ is finite. Conversely, if $\mathbb{F}(x)$ is algebraic over a finite field, then so is $\mathbb{F}\left(\varphi_{L, K}(x)\right)$, and $\varphi_{L, K}(x)$ is closed. By (1), $x$ is closed.

(3) Note that $x$ is closed in $X_{L}$ if and only if $x$ is closed in some neighborhood of the form Spec $A_{L}$ by (2). Thus it suffices to prove the assertion for $X=\operatorname{Spec} A$.

One can think of $A_{L}$ as the union of $A_{M}$ for all $M \in \mathcal{F}(L / K)$. If $\mathfrak{m} \subset A_{L}$ is a maximal ideal, then $\left(\mathfrak{m} \cap A_{M}\right)_{M \in \mathcal{F}(L / K)}$ is an element of $\lim _{M \in \mathcal{F}(L / K)} X_{M}^{0}$. Thus we have a natural map $f: X_{L}^{0} \rightarrow \lim _{M \in \mathcal{F}(L / K)} X_{M}^{0}$. From $\mathfrak{m}=\bigcup_{M \in \mathcal{F}(L / K)}\left(\mathfrak{m} \cap A_{M}\right)$ it follows that $f$ is injective.

If $\left(\mathfrak{m}_{M}\right)_{M \in \mathcal{F}(L / K)} \in \lim _{M \in \mathcal{F}(L / K)} X_{M}^{0}$, then $\mathfrak{p}:=\bigcup \mathfrak{m}_{M}$ is a prime ideal of $A_{L}$. Since $\mathfrak{m}_{M} \cap M^{\prime}=\mathfrak{m}_{M^{\prime}}$ holds for $M \supseteq M^{\prime}$, we have $\mathfrak{p} \cap M=\mathfrak{m}_{M}$. From (1) we see that $\mathfrak{p}$ is maximal, i.e. $\mathfrak{p} \in X_{L}^{0}$, and $f(\mathfrak{p})$ is equal to $\left(\mathfrak{m}_{M}\right)_{M \in \mathcal{F}(L / K)}$. Thus $f$ is surjective.

If $L / K$ is a Galois extension, then $L$ is the union of finite Galois extensions, so we may restrict to Galois extensions $M$.

In the rest of this section, let $\mathcal{M}$ be a subset of $X^{0}$ and $L$ a Galois extension of $K$ such that the Galois cover $\tilde{X}=X_{L}$ of $X$ is unramified over $\mathcal{M}$.

Definition 3.2. We define $Q(\tilde{X} / X, \mathcal{M})$ to be the set points of $\tilde{X}$ lying over $\mathcal{M}$. Using the notation in Proposition 3.1, $Q(\tilde{X} / X, \mathcal{M})=\varphi_{L, K}^{-1}(\mathcal{M})$.

If $M$ and $M^{\prime}$ are intermediate fields of $L / K$ which are Galois extensions of $K$ satisfying $M \supseteq M^{\prime}$, let $\pi_{M, M^{\prime}}: Q\left(X_{M} / X, \mathcal{M}\right) \rightarrow Q\left(X_{M^{\prime}} / X, \mathcal{M}\right)$ denote the restriction of $\varphi_{M, M^{\prime}}$.

Let $X^{\mathrm{ur}}$ denote the maximal unramified cover of $X$ and $X^{\mathrm{ur}, \mathrm{ab}}$ the maximal abelian unramified cover of $X$. We define $Q(X, \mathcal{M})$ and $Q^{\mathrm{ab}}(X, \mathcal{M})$ as $Q\left(X^{\mathrm{ur}} / X, \mathcal{M}\right)$ and $Q\left(X^{\mathrm{ur}, \mathrm{ab}} / X, \mathcal{M}\right)$, respectively.

\section{PROPOSITION 3.3.}

(1) The map $\pi_{L, M}$ is surjective.

(2) $Q(\tilde{X} / X, \mathcal{M})=\lim _{M \in \mathcal{G}(L / K)} Q\left(X_{M} / X, \mathcal{M}\right)$.

Proof. This follows immediately from the previous proposition.

When $[L: K]<\infty$, we endow $Q(\tilde{X} / X, \mathcal{M})$ with the discrete topology. In the general case, we endow $Q(\tilde{X} / X, \mathcal{M})$ with the topology as a projective limit, i.e. the topology induced by the product topology on $\prod_{M \in \mathcal{G}(L / K)} Q\left(X_{M} / X, \mathcal{M}\right)$. 
Since $\pi_{L, K}^{-1}(a)$ is a compact open set for any $a \in \mathcal{M}$, we see that $Q(\tilde{X} / X, \mathcal{M})$ is a locally compact space.

Notation 3.4. If $x$ is an element of $Q(\tilde{X} / X, \mathcal{M})$ we write $q(x)$ for $\# \mathbb{F}\left(\pi_{L, K}(x)\right)$ and $c(x)$ for char $\mathbb{F}(x)$. We may identify $c$ with the natural map $Q(\tilde{X} / X, \mathcal{M}) \rightarrow \operatorname{Spec} \mathbb{Z}$.

We denote by $s_{x}$ the Frobenius automorphism of $\tilde{X}$ associated to $x$, i.e. the unique element of $\operatorname{Aut}(\tilde{X} / X)$ such that $s_{x}(x)=x$ and $s_{x}^{*}(f) \equiv f^{q(x)} \bmod \mathfrak{m}_{x}$ for any $f \in \mathcal{O}_{\tilde{X}, x}$, where $\mathfrak{m}_{x}$ is the maximal ideal of $\mathcal{O}_{\tilde{X}, x}$.

We denote the corresponding element of $\operatorname{Gal}(L / K)$ by $F_{x}$.

Definition 3.5. Define the binary operation $\triangleright$ on $Q(\tilde{X} / X, \mathcal{M})$ by

$$
x \triangleright y:=s_{x}(y) .
$$

PROPOSITION 3.6.

(1) The pair $(Q(\tilde{X} / X, \mathcal{M})$, Aut $(\tilde{X} / X))$, together with the natural action and the map

$$
s_{L}: Q(\tilde{X} / X, \mathcal{M}) \rightarrow \operatorname{Aut}(\tilde{X} / X) ; x \mapsto s_{x},
$$

is a topological augmented quandle with the associated quandle operation $\triangleright$.

(2) For an intermediate Galois extension $M$ of $L / K$, the projection maps $\pi_{L, M}$ : $Q(\tilde{X} / X, \mathcal{M}) \rightarrow Q\left(X_{M} / X, \mathcal{M}\right)$ and $\pi_{L, M}^{\prime}: \operatorname{Aut}(\tilde{X} / X) \rightarrow \operatorname{Aut}\left(X_{M} / X\right)$ form a homomorphism of topological augmented quandles.

Proof. We will prove the assertions for finite Galois extensions $L$ over $K$. Then we see assertion (1) in the general case by taking the projective limit. For (2), noting that $Q\left(X_{M} / X, \mathcal{M}\right)=\lim _{L^{\prime} \in \mathcal{G}(L / K)} Q\left(X_{L^{\prime} \cap M} / X, \mathcal{M}\right)$, we can deduce the assertion that $\left(\pi_{L, M}, \pi_{L, M}^{\prime}\right)$ is a homomorphism of topological augmented quandles from the case of finite extensions.

So we assume that $L$ is a finite Galois extension of $K$. In this case all relevant spaces are endowed with discrete topology, so the continuity is obvious.

For (1), by the definition of Frobenius automorphism, we have $s_{x}(x)=x$ for any $x \in Q(\tilde{X} / X, \mathcal{M})$.

Let us show that $s_{g(x)}=g \circ s_{X} \circ g^{-1}$ holds for any $g \in \operatorname{Aut}(\tilde{X} / X)$ and $x \in Q(\tilde{X} / X, \mathcal{M})$. We set $x^{\prime}=g(x)$ and prove that $g \circ s_{X} \circ g^{-1}$ satisfies the conditions required for $s_{X^{\prime}}$. We have

$$
\begin{aligned}
g \circ s_{x} \circ g^{-1}\left(x^{\prime}\right) & =g\left(s_{x}(x)\right) \\
& =g(x)=x^{\prime} .
\end{aligned}
$$

For any $f \in \mathcal{O}_{\tilde{X}, x^{\prime}}$, we have $\left(g \circ s_{x} \circ g^{-1}\right)^{*} f \in \mathcal{O}_{\tilde{X}, x^{\prime}}$ from the above, and the assumption on $s_{x}$ implies that

$$
\begin{aligned}
\left(g \circ s_{x} \circ g^{-1}\right)^{*} f & =\left(g^{*}\right)^{-1}\left(s_{x}^{*}\left(g^{*}(f)\right)\right) \\
& =\left(g^{*}\right)^{-1}\left(\left(g^{*}(f)\right)^{q}+r\right),
\end{aligned}
$$

where $r$ is an element of $\mathfrak{m}_{x}$ and $q=q(x)=q\left(x^{\prime}\right)$. The right-hand side is equal to $f^{q}+\left(g^{*}\right)^{-1}(r)$, hence congruent to $f^{q}$ modulo $\mathfrak{m}_{x^{\prime}}$. Thus $g \circ s_{x} \circ g^{-1}$ is the Frobenius automorphism associated to $x^{\prime}$. 
For (2), we show that $F_{\pi_{L, M}(x)}=\left.F_{X}\right|_{M}$ holds for any $x \in Q(\tilde{X} / X, \mathcal{M})$. Let $g$ be the automorphism of $X_{M}$ associated to $\left.F_{X}\right|_{M}$. If $f \in \mathcal{O}_{X_{M}, \pi_{L, M}(x)}$, then it can also be considered as an element of $\mathcal{O}_{\tilde{X}, x}$ and therefore $F_{X}(f)$ is contained in $\mathcal{O}_{\tilde{X}, x} \cap M=\mathcal{O}_{X_{M}, \pi_{L, M}(x)}$. This shows that $g\left(\pi_{L, M}(x)\right)=\pi_{L, M}(x)$.

We have $F_{x}(f)-f^{q} \in \mathfrak{m}_{x} \cap \mathcal{O}_{X_{M}, \pi_{L, M}(x)}=\mathfrak{m}_{\pi_{L, M}(x)}$, so $g$ is the Frobenius automorphism associated to $\pi_{L, M}(x)$ and we have $F_{\pi_{L, M}(x)}=\left.F_{x}\right|_{M}$. This means $s_{M}$ 。 $\pi_{L, M}=\pi_{L, M}^{\prime} \circ s_{L}$. The actions are obviously compatible with the projection maps, so $\left(\pi_{L, M}, \pi_{L, M}^{\prime}\right)$ is a homomorphism of augmented quandles.

Remark 3.7. If $K$ is a knot in $\mathbb{R}^{3}$, its knot quandle $Q_{K}$ has a description analogous to the arithmetic case.

More generally, let us consider the following situation. Let $X$ be a path-connected, locally path-connected and locally simply connected topological space. Let $\varphi: \tilde{X} \rightarrow X$ be a regular (i.e. Galois) cover, $\left\{Y_{\lambda}\right\}_{\lambda \in \Lambda}$ a set of path-connected, locally path-connected and locally simply connected subsets of $X$ and $\left\{z_{\lambda}\right\}_{\lambda \in \Lambda}$ elements of $Z\left(\pi_{1}\left(Y_{\lambda}\right)\right)$. Note that we do not have to worry about the base point for $\pi_{1}\left(Y_{\lambda}\right)$ since we are considering an element in the center.

Then we can define a quandle operation on

$$
Q_{\text {top }}\left(\tilde{X} / X,\left\{Y_{\lambda}\right\},\left\{z_{\lambda}\right\}\right):=\coprod_{\lambda \in \Lambda} \pi_{0}\left(\varphi^{-1}\left(Y_{\lambda}\right)\right)
$$

in the following way. Let $Z_{1}$ and $Z_{2}$ be connected components of $\varphi^{-1}\left(Y_{\lambda}\right)$ and $\varphi^{-1}\left(Y_{\mu}\right)$, respectively. Let $m_{Z_{1}}$ be the deck transformation of $Z_{1} \rightarrow Y_{\lambda}$ corresponding to $s_{\lambda}$. There is a unique lift of $m_{Z_{1}}$ to a deck transformation of $\tilde{X} \rightarrow X$, which we denote by $\tilde{m}_{Z_{1}}$. Then $Z_{1} \triangleright Z_{2}=\tilde{m}_{Z_{1}}\left(Z_{2}\right)$. By choosing base points $p \in X$ and $p_{\lambda} \in Y_{\lambda}$ and paths connecting $p$ to $p_{\lambda}$, we can represent this quandle as in Example 2.4.

For a knot $K$, let $X$ be the knot complement $\mathbb{R}^{3} \backslash K, \tilde{X}$ the universal cover of $X$, and $\Sigma$ the boundary of a tubular neighborhood of $K$. Then $Q_{K}$ can be identified with $Q_{\text {top }}\left(\tilde{X} / X,\{\Sigma\},\left\{\right.\right.$ meridian\}). In fact, the knot quandle $Q_{K}$ of $K$ can be set-theoretically identified with $\pi_{0}\left(\varphi^{-1}(\Sigma)\right)$ by Theorem 16.1 of [J82]. It is easy to show that the quandle operations coincide.

For a prime $\mathfrak{p}$ in a number field $K$, let $X=\operatorname{Spec} \mathcal{O}_{K} \backslash\{\mathfrak{p}\}$ and $Y=\operatorname{Spec} K_{\mathfrak{p}}$. Then a direct analogue of knot quandles would be the limit of $Y \times_{X} \tilde{X}$ for connected étale covers $\tilde{X} \rightarrow X$. This does not work in a straightforward way, since there is no canonical choice for an analogue of the meridian, called a monodromy in [M12, Table (3.3)], and a monodromy will not be in the center. It might be possible to pursue the analogy by restricting covers in a certain way, or by considering generalization of quandles such as multiple conjugation quandles [I15].

Our quandle can be regarded as an analogue of $Q_{\text {top }}\left(\tilde{X} / X,\left\{Y_{\lambda}\right\},\left\{z_{\lambda}\right\}\right)$ where $Y_{\lambda}$ are simple loops and $z_{\lambda}$ are the positive generators of $\pi_{1}\left(Y_{\lambda}\right)$.

Proposition 3.8. Write $Q=Q(\tilde{X} / X, \mathcal{M})$ and $G=\operatorname{Aut}(\tilde{X} / X)$ and let $\left\{q_{a}\right\}_{a \in \mathcal{M}}$ be $a$ complete system of representatives of $G \backslash Q$, which is naturally in bijection with $\mathcal{M}$. Then $Q$ is naturally isomorphic to $Q\left(G,\left\{\overline{\left\langle s_{q_{a}}\right\rangle}\right\},\left\{s_{q_{a}}\right\}\right)$.

Proof. This follows from Proposition 2.9, since the stabilizer of $q_{a}$ is $\overline{\left\langle s_{q_{a}}\right\rangle}$. 
Definition 3.9. Let $X$ and $Y$ be topological spaces. We consider the set $C(X, Y)$ of continuous maps from $X$ to $Y$ with the compact-open topology. In other words, it has a sub-basis consisting of the sets

$$
O(C, U):=\{f \in C(X, Y) \mid f(C) \subseteq U\}
$$

for various compact subsets $C$ of $X$ and open subsets $U$ of $Y$.

If $X, Y$ and $Z$ are locally compact, it is known that $C(X, Y)$ is locally compact and that the evaluation map $C(X, Y) \times X \rightarrow Y$ and the composition map $C(Y, Z) \times C(X, Y) \rightarrow$ $C(X, Z)$ are continuous.

Proposition 3.10. Let $Q$ be the quandle $Q(\tilde{X} / X, \mathcal{M})$. Then the natural map $\gamma$. $\operatorname{Aut}(\tilde{X} / X) \rightarrow C(Q, Q)$ is continuous.

Proof. This follows from the continuity of the action and the following general fact: If $X, Y$ and $Z$ are locally compact spaces, a map $X \rightarrow C(Y, Z)$ is continuous if and only if the associated map $X \times Y \rightarrow Z$ is continuous.

\section{Reconstruction of arithmetic curves}

Now we restrict to the case of spectra of integer rings and study the problem of reconstruction.

Let $K$ be a number field, $\mathcal{O}_{K}$ the ring of integers in $K$, and $S$ and $\mathcal{M}$ disjoint sets of closed points of $\operatorname{Spec} \mathcal{O}_{K}$ with $\# S<\infty$. We take $\operatorname{Spec} \mathcal{O}_{K, S}=\operatorname{Spec} \mathcal{O}_{K} \backslash S$ as $X$.

\subsection{Reconstruction of the Galois group}

Let $L$ be a Galois extension of $K$ which is unramified over $\mathcal{M}$, and write $G$ for $\operatorname{Aut}\left(X_{L} / X\right)$ and $Q$ for $Q\left(X_{L} / X, \mathcal{M}\right)$.

Proposition 4.1. Assume that $\mathcal{M}$ has density one.

(1) The natural map $\gamma: G \rightarrow C(Q, Q)$ is injective. Hence it is a homeomorphism onto its image.

(2) The closure of $\operatorname{Inn}(Q)$ in $C(Q, Q)$ is equal to $\gamma(G)$.

Proof. (1) Let $g \neq \mathrm{id}_{X_{L}}$ be an element of $G$. Then there exists an intermediate finite Galois extension $M$ such that the induced automorphism $g_{M}$ of $M$ is not the identity. By the density theorem, there exists an element $a \in \mathcal{M}$ which decomposes completely in $M$. Then $\pi_{M, K}^{-1}(a)$ is a torsor over $\operatorname{Aut}\left(X_{M} / X\right)$, so the action of $g_{M}$ on this fiber is not trivial. Therefore $\gamma(g)$ is not the identity element.

The map $\gamma$ is a homeomorphism onto its image since it is continuous by Proposition 3.10 , injective as we just saw, $G$ is compact and $C(Q, Q)$ is Hausdorff.

(2) By the definition of the quandle operation, $\operatorname{Inn}(Q)$ is a subset of $\gamma(G)$. Let $g_{0}$ be any element of $G$. Then any neighborhood $V$ of $g_{0}$ contains a subset of the form $g_{0} \cdot \operatorname{Aut}\left(X_{L} / X_{M}\right)$ with $M$ a finite Galois extension of $K$. This is the set of elements of $G$ which induce the same element of $\operatorname{Aut}\left(X_{M} / X\right)$ as $g_{0}$. By the density theorem, $\left\{s_{y} \mid y \in\right.$ $\left.Q\left(X_{M} / X, \mathcal{M}\right)\right\}=\operatorname{Aut}\left(X_{M} / X\right)$ holds. Thus one can find $x \in Q$ such that $s_{X} \in V$.

It follows that $\operatorname{Inn}(Q)$ is dense in $\gamma(G)$. The latter is closed since $\gamma$ is continuous, $G$ is compact and $C(Q, Q)$ is Hausdorff. 
Remark 4.2. As can be seen from the proof, $\left\{s_{x} \mid x \in Q\right\}$ is already dense in $\gamma(G)$. It follows that $\gamma(G)$ is also equal to the closure of $\operatorname{Tr}(Q)$.

COROLlary 4.3. Assume that $\mathcal{M}$ has density one.

(1) As a topological group, $G=\operatorname{Aut}\left(X_{L} / X\right)$ can be recovered from the topological quandle $Q=Q\left(X_{L} / X, \mathcal{M}\right)$ as the closure $\overline{\operatorname{Inn}(Q)}$ of $\operatorname{Inn}(Q)$ in $C(Q, Q)$.

(2) The following three objects are the same: (a) $\overline{\operatorname{Inn}(Q)}$-orbits, (b) closures of $\operatorname{Inn}(Q)$ orbits, and $(c)$ inverse images of elements of $\mathcal{M}$.

Proof. (1) This is an immediate consequence of the proposition.

(2) Let $x$ be an element of $Q$. Since the action is continuous, we have $\overline{\operatorname{Inn}(Q)} \cdot x \subseteq$ $\overline{\operatorname{Inn}(Q) \cdot x}$. The set $\overline{\operatorname{Inn}(Q)} \cdot x$ contains $\operatorname{Inn}(Q) \cdot x$ and is closed since $\overline{\operatorname{Inn}(Q)} \cong \operatorname{Aut}\left(X_{L} / X\right)$ is compact. Thus $\overline{\operatorname{Inn}(Q)} \cdot x \supseteq \overline{\operatorname{Inn}(Q) \cdot x}$ holds.

Let us write $\pi$ for $\pi_{L, K}$. The inclusion $\operatorname{Inn}(Q) \cdot x \subseteq \pi^{-1}(\pi(x))$ is obvious, and since $\mathcal{M}$ is given the discrete topology, $\pi^{-1}(\pi(x))$ is closed and $\overline{\operatorname{Inn}(Q) \cdot x} \subseteq \pi^{-1}(\pi(x))$ holds. Let $y$ be an element of $\pi^{-1}(\pi(x))$. Then, for any finite Galois intermediate field $M$, the point $\pi_{L, M}(y)$ is in the $G$-orbit of $\pi_{L, M}(x)$. Thus $y$ is in the closure of $G \cdot x$, which is equal to $\overline{\operatorname{Inn}(Q) \cdot x}$.

Remark 4.4. We see from Corollary 4.3(2) that $\mathcal{M}$ as a set and the map $Q \rightarrow \mathcal{M}$ can be recovered from $Q$. Note that at this stage we do not know when and how the embedding of $\mathcal{M}$ into $\operatorname{Spec} \mathcal{O}_{K}$ can be recovered from $Q$.

Corollary 4.5. Assume that $\mathcal{M}$ has density one and let $K^{\mathrm{ab}}$ denote the maximal abelian extension of $K$. Then the topological quandle $Q\left(X_{L \cap K^{\mathrm{ab}}} / X, \mathcal{M}\right)$ can be described in terms of $Q$ as $Q / \overline{[\operatorname{Inn}(Q), \operatorname{Inn}(Q)]}$.

In particular, $Q^{\mathrm{ab}}(X, \mathcal{M})$ can be described in terms of $Q(X, \mathcal{M})$.

Proof. We have $\overline{[G, G]}=\overline{[\overline{\operatorname{Inn}(Q)}, \overline{\operatorname{Inn}(Q)}]}=\overline{[\operatorname{Inn}(Q), \operatorname{Inn}(Q)]}$, and hence the assertion follows.

\subsection{The case $S=\{\mathfrak{p}\} ; p$-adic logarithms and transcendency results}

Let us study the problem of reconstruction from $Q^{\mathrm{ab}}(X, \mathcal{M})$ in the special case where $S$ consists of one point $\mathfrak{p}$.

As in the case of reconstruction of curves from the arithmetic fundamental groups, it seems reasonable to require that the fundamental group be sufficiently big.

Let $Q$ and $G$ denote $Q^{\mathrm{ab}}(X, \mathcal{M})$ and $\pi_{1}^{\mathrm{ab}}(X)=\operatorname{Aut}\left(X^{\mathrm{ur}, \mathrm{ab}} / X\right)$, respectively.

PROPOSITION 4.6. If $\operatorname{Inn}(Q)$ is infinite, then char $\mathbb{F}(\mathfrak{p})$ is characterized as the unique prime number $p$ such that the p-part of the profinite group $\overline{\operatorname{Inn}(Q)}$ is infinite.

In particular, if $\mathcal{M}$ has density one and $G$ is infinite, then $p=\operatorname{char} \mathbb{F}(\mathfrak{p})$ and the rank of the p-part of $G$ can be recovered from $Q$.

Proof. By class field theory, there is an exact sequence

$$
1 \rightarrow \mathcal{O}_{K_{\mathfrak{p}}}^{\times} \overline{\mathcal{O}_{K}^{\times,+}} \rightarrow G \rightarrow \mathrm{Cl}^{+}(K) \rightarrow 1,
$$


where $K_{\mathfrak{p}}$ is the completion of $K$ at $\mathfrak{p}, \mathcal{O}_{K_{\mathfrak{p}}}$ its ring of integers, $\mathcal{O}_{K}^{\times,+}$is the group of totally positive units in $K, \overline{\mathcal{O}_{K}^{\times,+}}$is its closure in $\mathcal{O}_{K_{\mathfrak{p}}}$ and $\mathrm{Cl}^{+}(K)$ is the narrow class group of $K$. Thus char $\mathbb{F}(\mathfrak{p})$ is the unique prime number $p$ such that the $p$-part of $G$ is infinite.

By Proposition 3.10, $\overline{\operatorname{Inn}(Q)}$ is a closed subgroup of a quotient of $G$. So its $p$-part is finite if $p \neq \operatorname{char} \mathbb{F}(\mathfrak{p})$, and infinite if $p=\operatorname{char} \mathbb{F}(\mathfrak{p})$ and $\operatorname{Inn}(Q)$ is infinite.

If $\mathcal{M}$ has density one, then $G \cong \overline{\operatorname{Inn}(Q)}$ and the second assertion follows.

By the exact sequence above, the abelianized fundamental group $G$ can be linearized up to torsions using the $p$-adic logarithm function. In the proof of our main theorem, a key role is played by certain results on independence of $p$-adic logarithms of algebraic numbers. Let us collect the results from $p$-adic transcendental number theory that will be needed.

Let $k$ be a non-archimedean local field of characteristic zero. The power series $f(z):=$ $\sum_{n=1}^{\infty}(-1)^{n-1}(z-1)^{n} / n$ converges on a neighborhood of 1 in $k$. It can be extended to a function $\ln : \mathcal{O}_{k}^{\times} \rightarrow k$ by defining $\ln (z)$ to be $f\left(z^{N}\right) / N$ for a sufficiently divisible integer $N$. It has a local inverse map near zero given by the power series $\exp (z):=\sum_{n=0}^{\infty} z^{n} / n !$.

THEOREM 4.7. ( $p$-adic six-exponentials theorem [S66, Théorème 1]) Let $k$ be a nonarchimedean local field of characteristic zero. For positive integers $d$ and $l$ satisfying $d>l /(l-1)($ i.e. $d l>d+l)$, let $x_{1}, \ldots, x_{d}$ be elements of $k$ and $Y$ a free abelian subgroup of $k$ of rank $l$.

If $\exp \left(x_{i} y\right)$ converges and is algebraic over $\mathbb{Q}$ for any $i=1, \ldots, d$ and $y \in Y$, then $x_{1}, \ldots, x_{d}$ are linearly dependent over $\mathbb{Q}$.

This theorem is often used in the following form, hence the name.

COROLlary 4.8. For $1 \leq i \leq 2$ and $1 \leq j \leq 3$, let $\alpha_{i j} \in \mathcal{O}_{k}^{\times}$be algebraic over $\mathbb{Q}$. If the rows and columns of the matrix $M:=\left(\ln \alpha_{i j}\right)$ are linearly independent over $\mathbb{Q}$, then $M$ is of rank two (as a matrix over $k$ ).

Proof. Assume that $M$ is of rank one. Then one can find $x_{i}, y_{j} \in k$ such that $\ln \alpha_{i j}=x_{i} y_{j}$. The rank of $Y:=\left\langle y_{1}, y_{2}, y_{3}\right\rangle$ is three by the assumption that the columns of $M$ are linearly independent over $\mathbb{Q}$. The six-exponentials theorem implies that $x_{1}$ and $x_{2}$ are linearly dependent over $\mathbb{Q}$, but this contradicts the assumption that the rows of $M$ are linearly independent over $\mathbb{Q}$.

The six-exponentials theorem was generalized in the following form.

THEOREM 4.9. [W81, Théorème 2.1.p] Let $k$ be a non-archimedean local field of characteristic zero. For positive integers $d$ and l, let $\alpha_{i j} \in \mathcal{O}_{k}^{\times}$be algebraic over $\mathbb{Q}$ for $1 \leq i \leq d$ and $1 \leq j \leq l$.

Then the rank $r$ of $M:=\left(\ln \alpha_{i j}\right)$ satisfies $r \geq d \cdot \theta(M) /(1+\theta(M))$, where $\theta(M)$ is defined in [W81, §7].

(See [W81, Théorème 1.1.p] for a formulation similar to Theorem 4.7.)

We do not write down the definition of $\theta(M)$ here, but we remark that it is equal to $l / d$ if the following holds: for any non-zero vectors $\boldsymbol{x} \in \mathbb{Z}^{d}$ and $\boldsymbol{y} \in \mathbb{Z}^{l},{ }^{\mathrm{t}} \boldsymbol{x} M \boldsymbol{y} \neq 0$. In this case the theorem states that rank $M \geq d l /(d+l)$. 


\subsection{Rational and quadratic fields}

Now we furthermore assume that $K$ is either $\mathbb{Q}$ or a quadratic field. We denote the characteristic of $\mathbb{F}(\mathfrak{p})$ by $p$ and the rank of the $p$-part of $G$ by $R$. For a prime ideal $\mathfrak{l}$ of $K$, write $e(\mathfrak{l})$ and $f(\mathfrak{l})$ for the absolute ramification index and the degree of extension of $\mathbb{F}(\mathfrak{l})$ over the prime field, respectively. There are five cases.

(1) If $K=\mathbb{Q}$, then we have $G \cong \mathbb{Z}_{p}^{\times}$, so $R=1$.

(2) Let $K$ be a quadratic field.

(2-0) If $K$ is real and $e(\mathfrak{p})=f(\mathfrak{p})=1$, then $G$ is finite, so we will not consider this case.

(2-1) If $K$ is real and $(e(\mathfrak{p}), f(\mathfrak{p}))=(1,2)$ or $(2,1)$, then the unit group has rank one and $R=1$.

(2-2) If $K$ is complex and $e(\mathfrak{p})=f(\mathfrak{p})=1$, we again have $R=1$.

(2-3) If $K$ is complex and $(e(\mathfrak{p}), f(\mathfrak{p}))=(1,2)$ or $(2,1)$, we have $R=2$.

Example 4.10. Let us give an explicit description of the quandle $Q=Q^{\mathrm{ab}}(\operatorname{Spec} \mathbb{Z} \backslash$ $\{(p)\}, \mathcal{M})$ using Proposition 3.8. We identify the maximal ideals of $\mathbb{Z}$ with their positive generators, i.e. prime numbers. The fiber of $\pi: Q \rightarrow \mathcal{M}$ over $l \in \mathcal{M}$ can be identified with $\mathbb{Z}_{p}^{\times} / \overline{\langle l\rangle}$ under a choice of a point in $\pi^{-1}(l)$, so we have $Q=\bigsqcup_{l \in \mathcal{M}} \mathbb{Z}_{p}^{\times} / \overline{\langle l\rangle}$. Note that each fiber of $\pi$ is a finite set. (The same is true in the cases (2-0), (2-1) and (2-2). In the case (2-3), each fiber of $\pi$ is an infinite set.)

The quandle operation is given by $x \triangleright y=\pi(x) y$, where $\pi(x)$ is regarded as a rational prime and acts on each $\mathbb{Z}_{p}^{\times} / \overline{\langle l\rangle}$ by multiplication.

We can visualize the quandle $Q$ as a kind of slot machine. A pair of a reel and a button is associated to each $l \in \mathcal{M}$. When a button is pressed, reels rotate by certain angles. (Strictly speaking, $\mathbb{Z}_{p}^{\times} / \overline{\langle l\rangle}$ can be non-cyclic when $p=2$ and the word 'rotation' might be inappropriate.)

Now we state our main theorem.

THEOREM 4.11. (Main theorem) Let each of $K$ and $K^{\prime}$ be either $\mathbb{Q}$ or a quadratic field, $\mathfrak{p}$ and $\mathfrak{p}^{\prime}$ primes in $K$ and $K^{\prime}$ respectively, and $\mathcal{M}$ and $\mathcal{M}^{\prime}$ sets of primes with density one in $K$ and $K^{\prime}$, respectively.

Write $X=\operatorname{Spec} \mathcal{O}_{K} \backslash\{\mathfrak{p}\}$ and $Q=Q^{\mathrm{ab}}(X, \mathcal{M})$, with the natural maps $\varpi: Q \rightarrow X$ and $\rho: Q \rightarrow$ Spec $\mathbb{Z}$. Define $X^{\prime}, Q^{\prime}, \varpi^{\prime}$ and $\rho^{\prime}$ in the same way. Assume that $\pi_{1}^{\mathrm{ab}}(X)$ or $\pi_{1}^{\mathrm{ab}}\left(X^{\prime}\right)$ is infinite.

If $\varphi: Q \rightarrow Q^{\prime}$ is an isomorphism of topological quandles, then there exists an isomorphism $\sigma: K \rightarrow K^{\prime}$ which maps $\mathfrak{p}$ to $\mathfrak{p}^{\prime}$, and $\varphi$ commutes with $\rho$ and $\rho^{\prime}$.

Furthermore, except in the case where $K$ is real quadratic, $\sigma$ can be chosen so that $\varphi$ is compatible with the isomorphism $X \rightarrow X^{\prime}$ induced by $\sigma$.

Remark 4.12. A similar statement holds for $Q(X, \mathcal{M})$ and $Q\left(X^{\prime}, \mathcal{M}^{\prime}\right)$ by Corollary 4.5.

Let us begin the proof of the theorem. Write $p$ for the characteristic of $\mathbb{F}(\mathfrak{p})$, which can be recovered from $Q$ by Proposition 4.6 and is equal to the characteristic of $\mathbb{F}\left(\mathfrak{p}^{\prime}\right)$. Let $G=\pi_{1}^{\mathrm{ab}}(X)$ and write $G_{p}$ for its $p$-part. The latter is a $\mathbb{Z}_{p}$-module in a natural way, so let $V=G_{p} \otimes_{\mathbb{Z}_{p}} \mathbb{Q}_{p}$. Write $G^{\prime}, G_{p}^{\prime}$ and $V^{\prime}$ for the corresponding groups associated to $X^{\prime}$. 
The groups $G$ and $G^{\prime}$, the sets $\mathcal{M}$ and $\mathcal{M}^{\prime}$, and the maps $\pi: Q \rightarrow \mathcal{M}$ and $\pi^{\prime}: Q^{\prime} \rightarrow \mathcal{M}^{\prime}$ can be recovered from $Q$ and $Q^{\prime}$ by Corollary 4.3. (We are making a distinction between $\pi$ and $\varpi$ since $X$ is not yet reconstructed and sometimes we cannot recover the inclusion $\mathcal{M} \rightarrow X$.)

We also have maps $r: \mathcal{M} \rightarrow V$ and $r^{\prime}: \mathcal{M}^{\prime} \rightarrow V^{\prime}$ given by the quandle operation: for $a \in \mathcal{M}$, choose $x \in \pi^{-1}(a)$ and let $r(a)=\left(p\right.$-part of $\left.s_{x}\right) \otimes 1$. These are the maps induced by the reciprocity maps in class field theory.

Thus the theorem is reduced to the following.

THEOREM 4.13. Let the notation be as above and assume that $V$ is non-zero.

Let $\bar{\varphi}: \mathcal{M} \rightarrow \mathcal{M}^{\prime}$ be a bijection and $\psi: V \rightarrow V^{\prime}$ an isomorphism of $\mathbb{Q}_{p}$-vector spaces satisfying $r^{\prime} \circ \bar{\varphi}=\psi \circ r$.

Then there exists an isomorphism $\sigma: K \rightarrow K^{\prime}$ which maps $\mathfrak{p}$ to $\mathfrak{p}^{\prime}$, and the residue characteristics $c(\mathfrak{l})$ and $c(\bar{\varphi}(\mathfrak{l}))$ coincide for any $\mathfrak{l} \in \mathcal{M}$.

Furthermore, except in the real quadratic case, $\sigma$ can be chosen so that $\sigma(\mathfrak{l})=\bar{\varphi}(\mathfrak{l})$.

Note that, if we start with the description of $G$ by class field theory, there is a natural coordinate on $V$ given by the $p$-adic logarithm function. Here we start with $Q$, so there is no natural coordinate on $V$ and we cannot recover $\mathfrak{l}$ from $r(\mathfrak{l})$ directly. We can however take a number of prime ideals and compare the configurations of their images in $V$ and $V^{\prime}$.

If $K$ is a number field and $\mathfrak{p}$ is a prime in $K$, we denote the localization of $\mathcal{O}_{K}$ at $\mathfrak{p}$ by $\mathcal{O}_{K, \mathfrak{p}}$ and the composite map $\mathcal{O}_{K, \mathfrak{p}}^{\times} \rightarrow \mathcal{O}_{K_{\mathfrak{p}}}^{\times} \stackrel{\ln }{\rightarrow} K_{\mathfrak{p}}$ by $\ln ^{\mathfrak{p}}$. (We have to be a little careful about where we take the logarithms.)

The following fact is easy to see.

LEMMA 4.14. Let $k$ be a non-archimedean local field of characteristic zero, and $\alpha_{1}, \ldots, \alpha_{n}$ elements of $\mathcal{O}_{k}^{\times}$. Then $\ln \alpha_{1}, \ldots, \ln \alpha_{n}$ are linearly independent over $\mathbb{Q}$ if and only if $\alpha_{1}, \ldots, \alpha_{n}$ are multiplicatively independent.

In particular, if $K$ is a number field, $\mathfrak{p}$ is a prime in $K$, and $\alpha_{1}, \ldots, \alpha_{n}$ are elements of $\mathcal{O}_{K, \mathfrak{p}}^{\times}$such that the ideals $\alpha_{1} \mathcal{O}_{K}, \ldots, \alpha_{n} \mathcal{O}_{K}$ are multiplicatively independent, then $\ln ^{\mathfrak{p}} \alpha_{1}, \ldots, \ln ^{\mathfrak{p}} \alpha_{n}$ are linearly independent over $\mathbb{Q}$.

Denote by $R$ the dimension of $V$, which is equal to the dimension of $V^{\prime}$.

\subsection{Case $R=1$}

Let $\mathfrak{l}_{1}$ and $\mathfrak{l}_{2}$ be elements of $\mathcal{M}$. If $V$ is one-dimensional, the quantity $r\left(\mathfrak{l}_{2}\right) / r\left(\mathfrak{l}_{1}\right) \in \mathbb{Q}_{p}$ is a well-defined invariant in the sense that if $\bar{\varphi}\left(\mathfrak{l}_{1}\right)=\mathfrak{l}_{1}^{\prime}$ and $\bar{\varphi}\left(\mathfrak{l}_{2}\right)=\mathfrak{l}_{2}^{\prime}$ then $r\left(\mathfrak{l}_{2}\right) / r\left(\mathfrak{l}_{1}\right)=$ $r^{\prime}\left(\mathfrak{l}_{2}^{\prime}\right) / r^{\prime}\left(\mathfrak{l}_{1}^{\prime}\right)$ holds. Let us express $r\left(\mathfrak{l}_{2}\right) / r\left(\mathfrak{l}_{1}\right)$ in terms of $p$-adic logarithms.

(1) If $K=\mathbb{Q}$, then the image of $\mathfrak{l} \in \mathcal{M}$ in $G$ can be identified with $N(\mathfrak{l})=c(\mathfrak{l})$ under the identification of $G$ with $\mathbb{Z}_{p}^{\times}$. Thus

$$
r\left(\mathfrak{l}_{2}\right) / r\left(\mathfrak{l}_{1}\right)=\ln ^{(p)} N\left(\mathfrak{l}_{2}\right) / \ln ^{(p)} N\left(\mathfrak{l}_{1}\right) .
$$

(2-1) $K$ is real and $e(\mathfrak{p}) f(\mathfrak{p})=2$. Let $\gamma$ be a unit which is not a root of unity. Then $V=\left(\left(\mathcal{O}_{K_{\mathfrak{p}}}^{\times}\right)_{p} \otimes_{\mathbb{Z}_{p}} \mathbb{Q}_{p}\right) / \mathbb{Q}_{p} \gamma_{p}$, where $\gamma_{p}$ is the $p$-component of $\gamma$ in $\mathcal{O}_{K_{\mathfrak{p}}}^{\times}$. The $p$ adic logarithm function induces $\left(\mathcal{O}_{K_{\mathfrak{p}}}^{\times}\right)_{p} \otimes_{\mathbb{Z}_{p}} \mathbb{Q}_{p} \cong K_{\mathfrak{p}}$, and satisfies $\ln ^{\mathfrak{p}} \bar{z}=\overline{\ln ^{\mathfrak{p}} z}$ since 
$\overline{\mathfrak{p}}=\mathfrak{p}$. Here $\bar{z}$ is the conjugate of $z \in K_{\mathfrak{p}}$ over $\mathbb{Q}_{p}$. If $K=\mathbb{Q}(\sqrt{m})$, an isomorphism between $K_{\mathfrak{p}}$ and $\mathbb{Q}_{p}^{2}$ as $\mathbb{Q}_{p}$-vector spaces is given by $z \mapsto(z+\bar{z},(z-\bar{z}) / \sqrt{m})$. Composing this map with $\ln ^{\mathfrak{p}}$, we obtain a map $\alpha \mapsto\left(\ln ^{\mathfrak{p}} N(\alpha),\left(\ln ^{\mathfrak{p}} \alpha \bar{\alpha}^{-1}\right) / \sqrt{m}\right)$. This maps $\gamma$ to $\left(0,2 \ln ^{\mathfrak{p}} \gamma / \sqrt{m}\right)$. Thus $\ln ^{\mathfrak{p}} N(\alpha)=\ln ^{(p)} N(\alpha)$ gives a linear coordinate of the image of $\alpha$ in $V$.

Now let $\mathfrak{l}_{1}$ and $\mathfrak{l}_{2}$ be elements of $\mathcal{M}$. One can find a positive integer $n$ such that $\mathfrak{l}_{1}^{n}$ and $\mathfrak{l}_{2}^{n}$ are principal ideals generated by totally positive numbers $\alpha_{1}$ and $\alpha_{2}$. Then we have

$$
r\left(\mathfrak{l}_{2}\right) / r\left(\mathfrak{l}_{1}\right)=\ln ^{(p)} N\left(\alpha_{2}\right) / \ln ^{(p)} N\left(\alpha_{1}\right)=\ln ^{(p)} N\left(\mathfrak{l}_{2}\right) / \ln ^{(p)} N\left(\mathfrak{l}_{1}\right) .
$$

$(2-2) K$ is complex and $e(\mathfrak{p}) f(\mathfrak{p})=1$. Then $V=\left(\mathcal{O}_{K_{\mathfrak{p}}}^{\times}\right)_{p} \otimes_{\mathbb{Z}_{p}} \mathbb{Q}_{p}$. Let $\mathfrak{l}_{1}$ and $\mathfrak{l}_{2}$ be elements of $\mathcal{M}$. One can find a positive integer $n$ such that $\mathfrak{l}_{1}^{n}$ and $\mathfrak{l}_{2}^{n}$ are generated by $\alpha_{1}$ and $\alpha_{2}$, and then we have

$$
r\left(\mathfrak{l}_{2}\right) / r\left(\mathfrak{l}_{1}\right)=\ln ^{\mathfrak{p}} \alpha_{2} / \ln ^{\mathfrak{p}} \alpha_{1} .
$$

In any case, one can take any number of prime ideals $\mathfrak{l}_{i}(i=1,2, \ldots)$ from $\mathcal{M}$ with different residue characteristics and with $e\left(\mathfrak{l}_{i}\right)=f\left(\mathfrak{l}_{i}\right)=1$ since $\mathcal{M}$ has density one. Let $\mathfrak{l}_{i}^{\prime}$ be the corresponding elements of $\mathcal{M}^{\prime}$.

First consider the case where $K$ is of type (2-2). Take a positive number $n$ such that $\mathfrak{l}_{i}^{n}$ are principal and let $\alpha_{i}$ denote generators.

If $K^{\prime}$ is also of type (2-2), let $\alpha_{1}^{\prime}, \alpha_{2}^{\prime}$ and $\alpha_{3}^{\prime}$ be defined in the same way. If $K \cong K^{\prime}$, identify them by the isomorphism $\sigma$ which maps $\mathfrak{p}$ to $\mathfrak{p}^{\prime}$ and write $\mathfrak{P}$ for $\mathfrak{p}$. If $K \not K^{\prime}$, then $K K^{\prime}$ would be of degree four over $\mathbb{Q}$. Let $\mathfrak{P}$ be the prime ideal in $K K^{\prime}$ over $\mathfrak{p}$ and $\mathfrak{p}^{\prime}$. In either case, the logarithms at $\mathfrak{p}$ and $\mathfrak{p}^{\prime}$ can be regarded as the logarithms at $\mathfrak{P}$. If $K^{\prime}$ were of type (1) or (2-1), write $\alpha_{i}^{\prime}=N\left(\mathfrak{l}_{i}^{\prime}\right)$. In this case the logarithm at $(p)$ is equal to the logarithm at $\mathfrak{p}$. Write $\mathfrak{P}$ for $\mathfrak{p}$.

In any case, we have

$$
\operatorname{rank}\left(\begin{array}{lll}
\ln ^{\mathfrak{P}} \alpha_{1} & \ln ^{\mathfrak{P}} \alpha_{2} & \ln ^{\mathfrak{P}} \alpha_{3} \\
\ln ^{\mathfrak{P}} \alpha_{1}^{\prime} & \ln ^{\mathfrak{P}} \alpha_{2}^{\prime} & \ln ^{\mathfrak{P}} \alpha_{3}^{\prime}
\end{array}\right)=1 .
$$

By Corollary 4.8 the rows or the columns must be linearly dependent over $\mathbb{Q}$. Since $\mathfrak{l}_{i}$ are multiplicatively independent, the columns are independent by Lemma 4.14. Thus $\ln ^{\mathfrak{P}} \alpha_{1}$ and $\ln ^{\mathfrak{P}} \alpha_{1}^{\prime}$ must be dependent over $\mathbb{Q}$, and $\alpha_{1}$ and $\alpha_{1}^{\prime}$ are multiplicatively dependent. It follows that $\alpha_{1}^{m}$ is contained in $K^{\prime}$ for some $m \neq 0$. We see from $e\left(\mathfrak{l}_{1}\right)=f\left(\mathfrak{l}_{1}\right)=1$ that $\alpha_{1}^{m}$ generates $K$ over $\mathbb{Q}$, so $K^{\prime} \supseteq K$, hence $K=K^{\prime}$, holds.

The dependence of rows over $\mathbb{Q}$ implies that $\mathfrak{l}_{i}$ and $\mathfrak{l}_{i}^{\prime}$ are multiplicatively dependent, hence that $\mathfrak{l}_{i}=\mathfrak{l}_{i}^{\prime}$. Since $V$ is a one-dimensional vector space, $\psi$ is the identity map (under the identification by $\sigma)$. Since $\mathcal{M} \rightarrow\left(\mathcal{O}_{K_{\mathfrak{p}}}^{\times}\right)_{p} \otimes_{\mathbb{Z}_{p}} \mathbb{Q}_{p}$ is always injective and $V$ can be identified with $\left(\mathcal{O}_{K_{\mathfrak{p}}}^{\times}\right)_{p} \otimes_{\mathbb{Z}_{p}} \mathbb{Q}_{p}$ in this case, we see that $\bar{\varphi}$ is the identity map.

Next assume that $K$ is of type (1) or (2-1). Then $K^{\prime}$ is also of type (1) or (2-1). We show that the map $c$ and the field $K$ can be recovered from $r: \mathcal{M} \rightarrow V$. The residue characteristic $c\left(\mathfrak{l}_{1}\right)$ is the unique rational prime number $l_{1}$ such that

$$
r\left(\mathfrak{l}_{2}\right) / r\left(\mathfrak{l}_{1}\right) \equiv \ln ^{(p)} l_{2} / \ln ^{(p)} l_{1} \bmod \mathbb{Q}^{\times}
$$

and

$$
r\left(\mathfrak{l}_{3}\right) / r\left(\mathfrak{l}_{1}\right) \equiv \ln ^{(p)} l_{3} / \ln ^{(p)} l_{1} \bmod \mathbb{Q}^{\times}
$$


hold for certain primes $l_{2}$ and $l_{3}$. In fact, these relations imply that

$$
\operatorname{rank}\left(\begin{array}{ccc}
\ln ^{(p)} N\left(\mathfrak{l}_{1}\right) & \ln ^{(p)} N\left(\mathfrak{l}_{2}\right) & \ln ^{(p)} N\left(\mathfrak{l}_{3}\right) \\
\ln ^{(p)} l_{1} & a \cdot \ln ^{(p)} l_{2} & b \cdot \ln ^{(p)} l_{3}
\end{array}\right)=1
$$

for some rational numbers $a$ and $b$. Since we chose $\mathfrak{l}_{i}$ to have distinct residue characteristics, the columns are independent over $\mathbb{Q}$. By Corollary 4.8 , the rows are dependent, and $N\left(\mathfrak{l}_{1}\right)$ is a power of $l_{1}$.

The set $\left\{l \in \operatorname{Spec} \mathbb{Z} \mid \# c^{-1}(l)=2\right\}$ is of density one-half (respectively empty) if $K$ is quadratic (respectively $K=\mathbb{Q}$ ), and this set determines $K$.

\subsection{Case $R=2$}

This is the case where $K$ is complex and $e(\mathfrak{p}) f(\mathfrak{p})=2$.

We provide two proofs. The first one makes use of Theorem 4.9 and is somewhat more direct. The second one is based on a characterization of a special subspace $\mathbb{Q}_{p} \subset V \cong K_{\mathfrak{p}}$ and only uses Corollary 4.8 .

First proof. In $K K^{\prime}$, let $\mathfrak{P}$ be a prime over $\mathfrak{p}$ and $\mathfrak{p}^{\prime}$.

Let $\mathcal{M}_{1}=\{\mathfrak{l} \in \mathcal{M} \mid e(\mathfrak{l}) f(\mathfrak{l})=1, \overline{\mathfrak{l}} \in \mathcal{M}\}$ and $\mathfrak{l}$ an element of $\mathcal{M}_{1}$. Let $n$ be a positive integer such that $\mathfrak{l}^{n}$ is principal, generated by $\alpha$. Then $\left\{\ln ^{\mathfrak{p}} \alpha, \ln ^{\mathfrak{p}} \bar{\alpha}\right\}$ spans $K_{\mathfrak{p}}$ over $\mathbb{Q}_{p}$. In fact, if not, we would have $\ln ^{\mathfrak{p}} \alpha=z \cdot \ln ^{\mathfrak{p}} \bar{\alpha}$ for some $z \in \mathbb{Q}_{p}$. Conjugating, we also have $\ln ^{\mathfrak{p}} \bar{\alpha}=z \cdot \ln ^{\mathfrak{p}} \alpha$ and so $z$ must be \pm 1 . This contradicts Lemma 4.14.

Let $\mathfrak{l}^{\prime}=\bar{\varphi}(\mathfrak{l})$ and $\mathfrak{l}^{\prime \prime}=\bar{\varphi}(\overline{\mathfrak{l}})$. Assume that both $\left\{\mathfrak{l}, \overline{\mathfrak{l}}, \mathfrak{l}^{\prime}\right\}$ and $\left\{\mathfrak{l}, \overline{\mathfrak{l}}, \mathfrak{l}^{\prime \prime}\right\}$ are multiplicatively dependent in the ideal group of $K K^{\prime}$. Then some powers of $\mathfrak{l}^{\prime}$ and $\mathfrak{l}^{\prime \prime}$ are contained in $\langle\mathfrak{l}, \overline{\mathfrak{l}}\rangle$, and so $\mathfrak{l}^{\prime}$ and $\mathfrak{l}^{\prime \prime}$ lie over $c(\mathfrak{l})$. If $K \not K^{\prime}$, there would be four primes in $K K^{\prime}$ over $c(\mathfrak{l})$, namely $\mathfrak{l}_{1}:=\mathfrak{l}+\mathfrak{l}^{\prime}, \mathfrak{l}_{2}:=\mathfrak{l}+\mathfrak{l}^{\prime \prime}, \mathfrak{l}_{3}:=\overline{\mathfrak{l}}+\mathfrak{l}^{\prime}$ and $\mathfrak{l}_{4}:=\overline{\mathfrak{l}}+\mathfrak{l}^{\prime \prime}$, and $\mathfrak{l}^{\prime}=\mathfrak{l}_{1} \mathfrak{l}_{3}$ is not in the $\mathbb{Q}$-span of $\mathfrak{l}=\mathfrak{l}_{1} \mathfrak{l}_{2}$ and $\overline{\mathfrak{l}}=\mathfrak{l}_{3} \mathfrak{l}_{4}$. Thus $K=K^{\prime}$ and $\left\{\mathfrak{l}^{\prime}, \mathfrak{l}^{\prime \prime}\right\}=\{\mathfrak{l}, \overline{\mathfrak{l}}\}$. Since $\{r(\mathfrak{l}), r(\overline{\mathfrak{l}})\}$ spans $V \cong K_{\mathfrak{p}}$ over $\mathbb{Q}_{p}, \psi$ is either the identity or the conjugation map, and hence similarly for $\bar{\varphi}$.

Thus we suppose that either $\{\mathfrak{l}, \overline{\mathfrak{l}}, \bar{\varphi}(\mathfrak{l})\}$ or $\{\mathfrak{l}, \overline{\mathfrak{l}}, \bar{\varphi}(\overline{\mathfrak{l}})\}$ is multiplicatively independent for any $\mathfrak{l} \in \mathcal{M}_{1}$, and draw a contradiction.

We will apply Theorem 4.9 with $d=3$ and $l=7$. Choose $\mathfrak{l}_{1}, \ldots, \mathfrak{l}_{7} \in \mathcal{M}_{1}$ in the following way. Let $\mathfrak{l}_{1}$ be an element of $\mathcal{M}_{1}$ and write $\mathfrak{l}_{1}^{\prime}=\bar{\varphi}\left(\mathfrak{l}_{1}\right)$. From the above, we may assume that $\mathfrak{l}_{1}, \overline{\mathfrak{l}}_{1}$ and $\mathfrak{l}_{1}^{\prime}$ are multiplicatively independent. When $\mathfrak{l}_{1}, \ldots, \mathfrak{l}_{k-1}$ are chosen, let $\mathfrak{l}_{k}$ be an element of $\mathcal{M}_{1}$ such that, denoting $\bar{\varphi}\left(\mathfrak{l}_{k}\right)$ by $\mathfrak{l}_{k}^{\prime}$, the residue characteristics $c\left(\mathfrak{l}_{k}\right)$ and $c\left(\mathfrak{l}_{k}^{\prime}\right)$ are both different from any of $c\left(\mathfrak{l}_{i}\right)$ and $c\left(\mathfrak{l}_{i}^{\prime}\right), 1 \leq i<k$, and that $\mathfrak{l}_{k}, \overline{\mathfrak{l}}_{k}$ and $\mathfrak{l}_{k}^{\prime}$ are multiplicatively independent. We can carry this out since $\mathcal{M}$ is assumed to be of density one.

Let $n$ be a positive integer such that $\mathfrak{l}_{i}^{n}=\left(\alpha_{i}\right)$ and $\left(\mathfrak{l}_{i}^{\prime}\right)^{n}=\left(\beta_{i}\right)$ holds. Then we have

$$
\operatorname{rank}\left(\begin{array}{llll}
\ln ^{\mathfrak{P}} \alpha_{1} & \ln ^{\mathfrak{P}} \alpha_{2} & \ldots & \ln ^{\mathfrak{P}} \alpha_{7} \\
\ln ^{\mathfrak{P}} \bar{\alpha}_{1} & \ln ^{\mathfrak{P}} \bar{\alpha}_{2} & \ldots & \ln ^{\mathfrak{P}} \bar{\alpha}_{7} \\
\ln ^{\mathfrak{P}} \beta_{1} & \ln ^{\mathfrak{P}} \beta_{2} & \ldots & \ln ^{\mathfrak{P}} \beta_{7}
\end{array}\right) \leq 2 .
$$

In fact, since $K_{\mathfrak{p}}$ is of dimension two over $\mathbb{Q}_{p}$, the entries of the first row can be written as a $\mathbb{Q}_{p}$-linear combination of two of them, say $\ln ^{\mathfrak{P}} \alpha_{i}$ and $\ln ^{\mathfrak{P}} \alpha_{j}$. The other rows are obtained by applying $\mathbb{Q}_{p}$-linear maps to the entries of the first row. Thus columns of the matrix can be written as a $\mathbb{Q}_{p}$-linear combination of the $i$ th and $j$ th columns. 
The entries are linearly independent over $\mathbb{Q}$ : The prime ideals (in $K$ or $K^{\prime}$ ) corresponding to entries in different columns belong to different residue characteristics, so a linear relation of entries implies a linear relation of entries in a column. But we assumed that entries in a column are linearly independent.

Thus the condition stated after Theorem 4.9 is satisfied, and the theorem asserts that the rank is at least $3 \cdot 7 /(3+7)>2$, a contradiction.

Second proof. We characterize the subspace $\mathbb{Q}_{p}$ of $V \cong K_{\mathfrak{p}}$ in terms of the map $r: \mathcal{M} \rightarrow V$.

LEMMA 4.15. Let us identify $V$ with $K_{\mathfrak{p}}$.

(1) The following conditions are equivalent for a one-dimensional $\mathbb{Q}_{p}$-subspace $W$ of $V$.

(a) $W=\mathbb{Q}_{p}$.

(b) For any finite subset $E$ of $\mathcal{M}$, the set $P:=\left\{\left\{\mathfrak{l}, \mathfrak{l}^{\prime}\right\} \subset \mathcal{M} \backslash E \mid r(\mathfrak{l})+r\left(\mathfrak{l}^{\prime}\right) \in\right.$ $\left.W, \mathfrak{l} \neq \mathfrak{l}^{\prime}\right\}$ is non-empty (hence infinite).

(c) The set $P:=\left\{\left\{\mathfrak{l}, \mathfrak{l}^{\prime}\right\} \subset \mathcal{M} \mid r(\mathfrak{l})+r\left(\mathfrak{l}^{\prime}\right) \in W, \mathfrak{l} \neq \mathfrak{l}^{\prime}\right\}$ has at least three elements.

(2) If $\mathfrak{l} \in \mathcal{M}$ is such that $r(\mathfrak{l}) \notin \mathbb{Q}_{p}$, there is at most one $\mathfrak{l}^{\prime} \in \mathcal{M}$ such that $r(\mathfrak{l})+r\left(\mathfrak{l}^{\prime}\right) \in \mathbb{Q}_{p}$, namely $\mathfrak{l}^{\prime}=\overline{\mathfrak{l}}$ (assuming it is contained in $\mathcal{M}$ ).

Proof. (1) (a) $\Rightarrow$ (b) We have $r(\overline{\mathfrak{l}})=\overline{r(\mathfrak{l})}$, so $r(\mathfrak{l})+r(\overline{\mathfrak{l}}) \in \mathbb{Q}_{p}$ holds for any $\mathfrak{l} \in \mathcal{M}$. Since $\mathcal{M}$ has density one, one can find $\mathfrak{l} \in \mathcal{M} \backslash(E \cup \bar{E})$ such that $\mathfrak{l} \neq \overline{\mathfrak{l}}$.

(b) $\Rightarrow$ (c) This is obvious.

(c) $\Rightarrow$ (a) Let $\left\{\mathfrak{l}_{1}, \mathfrak{l}_{2}\right\},\left\{\mathfrak{l}_{3}, \mathfrak{l}_{4}\right\}$ and $\left\{\mathfrak{l}_{5}, \mathfrak{l}_{6}\right\}$ be distinct sets of two primes such that $r\left(\mathfrak{l}_{1}\right)+$ $r\left(\mathfrak{l}_{2}\right), r\left(\mathfrak{l}_{3}\right)+r\left(\mathfrak{l}_{4}\right)$ and $r\left(\mathfrak{l}_{5}\right)+r\left(\mathfrak{l}_{6}\right)$ are contained in $W$. Write $z_{i}$ for $r\left(\mathfrak{l}_{i}\right)=\left(\ln ^{\mathfrak{p}} \alpha_{i}\right) / n$, where $l_{i}^{n}$ is principal with generator $\alpha_{i}$. Note that $z, w \in K_{\mathfrak{p}}$ are linearly dependent over $\mathbb{Q}_{p}$ if and only if

$$
\operatorname{rank}\left(\begin{array}{cc}
z & w \\
\bar{z} & \bar{w}
\end{array}\right) \leq 1
$$

Thus we have

$$
\operatorname{rank}\left(\begin{array}{lll}
z_{1}+z_{2} & z_{3}+z_{4} & z_{5}+z_{6} \\
\bar{z}_{1}+\bar{z}_{2} & \bar{z}_{3}+\bar{z}_{4} & \bar{z}_{5}+\bar{z}_{6}
\end{array}\right)=1 \text {. }
$$

Assume that $a\left(z_{1}+z_{2}\right)+b\left(z_{3}+z_{4}\right)+c\left(z_{5}+z_{6}\right)=0$ holds for $a, b, c \in \mathbb{Z}$. Then $\left(\mathfrak{l}_{1} \mathfrak{l}_{2}\right)^{a}\left(\mathfrak{l}_{3} \mathfrak{l}_{4}\right)^{b}\left(\mathfrak{l}_{5} \mathfrak{l}_{6}\right)^{c}=(1)$ holds. Write $k$ for $\#\left\{\mathfrak{l}_{1}, \ldots, \mathfrak{l}_{6}\right\}$, and we have a relation of the form $a \boldsymbol{v}_{1}+b \boldsymbol{v}_{2}+c \boldsymbol{v}_{3}=\boldsymbol{o}$, where $\boldsymbol{v}_{i} \in \mathbb{Z}^{k}$ are pairwise distinct vectors with two entries equal to 1 and the other entries 0 . Then it is easy to see that $a=b=c=0$, and the columns of the above matrix are linearly independent over $\mathbb{Q}$.

Thus by Corollary 4.8 the rows are linearly dependent over $\mathbb{Q}$. It follows that $\mathfrak{l}_{1} \mathfrak{l}_{2}=\overline{\mathfrak{l}}_{1} \overline{\mathfrak{l}}_{2}$, and $z_{1}+z_{2} \in \mathbb{Q}_{p}$ holds.

(2) If $r(\mathfrak{l})+r\left(\mathfrak{l}^{\prime}\right) \in \mathbb{Q}_{p}$, then $r(\mathfrak{l})+r\left(\mathfrak{l}^{\prime}\right)=r(\overline{\mathfrak{l}})+r\left(\overline{\mathfrak{l}^{\prime}}\right)$ holds. It follows that $\mathfrak{l} \mathfrak{l}^{\prime}=\overline{\mathfrak{l}} \overline{\mathfrak{l}^{\prime}}$. Assuming that $r(\mathfrak{l}) \notin \mathbb{Q}_{p}, \mathfrak{l} \neq \overline{\mathfrak{l}}$ and hence $\mathfrak{l}^{\prime}=\overline{\mathfrak{l}}$ holds.

Remark 4.16. In Lemma 4.15(1), we also have the following sufficient condition: the set $\{\mathfrak{l} \in \mathcal{M} \mid r(\mathfrak{l}) \in W\}$ is infinite (or has at least three elements).

If we assume that $\mathcal{M}$ contains infinitely many (or at least three) primes $\mathfrak{l}$ with $e(\mathfrak{l}) f(\mathfrak{l})$ $=2$ (or equivalently $\mathfrak{l}=\overline{\mathfrak{l}}$ ), it is also a necessary condition.

By this lemma the subspace $W$ corresponding to $\mathbb{Q}_{p}$ can be recovered from $r: \mathcal{M} \rightarrow V$. The set $\mathcal{M} \cap \overline{\mathcal{M}}$ can be characterized as the set of $\mathfrak{l} \in \mathcal{M}$ satisfying the condition that 
either $r(\mathfrak{l}) \in W$ holds or there exists $\mathfrak{l}^{\prime} \in \mathcal{M}$ such that $r(\mathfrak{l})+r\left(\mathfrak{l}^{\prime}\right) \in W$. For $\mathfrak{l} \in \mathcal{M} \cap \overline{\mathcal{M}}$, its conjugate $\overline{\mathfrak{l}}$ is characterized as follows: If $r(\mathfrak{l}) \in W$, then $\overline{\mathfrak{l}}=\mathfrak{l}$. If not, $\overline{\mathfrak{l}}$ is the unique $\mathfrak{l}^{\prime} \in \mathcal{M}$ such that $r(\mathfrak{l})+r\left(\mathfrak{l}^{\prime}\right) \in W$.

Thus for $\mathfrak{l}_{1}, \mathfrak{l}_{2} \in \mathcal{M} \cap \overline{\mathcal{M}}$,

$$
\left(r\left(\mathfrak{l}_{2}\right)+r\left(\overline{\mathfrak{l}}_{2}\right)\right) /\left(r\left(\mathfrak{l}_{1}\right)+r\left(\overline{\mathfrak{l}}_{1}\right)\right)=\ln ^{(p)} N\left(\mathfrak{l}_{2}\right) / \ln ^{(p)} N\left(\mathfrak{l}_{1}\right)
$$

was recovered from $r: \mathcal{M} \rightarrow V$.

As in the case of type (1) or (2-1), we can recover the residue characteristics $c$ : $\mathcal{M} \cap \overline{\mathcal{M}} \rightarrow$ Spec $\mathbb{Z}$ and hence a set of rational primes of density one-half which split in $K$. Thus $K$ is determined, and one can find an isomorphism of $K$ and $K^{\prime}$. The isomorphism $\psi$ induces the identity map on $W$. If $\mathfrak{l} \in \mathcal{M} \cap \overline{\mathcal{M}}$ is such that $r(\mathfrak{l}) \notin W$, then $c(\bar{\varphi}(\mathfrak{l}))=c(\mathfrak{l})$, so $\bar{\varphi}(\mathfrak{l})$ is either $\mathfrak{l}$ or $\overline{\mathfrak{l}}$. Thus, choosing the other isomorphism of $K$ and $K^{\prime}$ if necessary, we may assume that $\bar{\varphi}(\mathfrak{l})=\mathfrak{l}$. Now the linear map $\psi: V \rightarrow V^{\prime}$ is the identity map, and so is $\mathcal{M} \rightarrow \mathcal{M}^{\prime}$ since $r$ is injective.

Remark 4.17. It is possible to ask whether analogous results hold for knots. As in the last paragraph of Remark 3.7, let $X$ be a knot complement (in $\mathbb{R}^{3}$ or a general 3-manifold), $\tilde{X}$ its universal cover, $\left\{Y_{\lambda}\right\}_{\lambda \in \Lambda}$ a set of simple loops in $X$ and $z_{\lambda} \in \pi_{1}\left(Y_{\lambda}\right)$ the positive generators. How much can we recover from $Q_{\text {top }}\left(\tilde{X} / X,\left\{Y_{\lambda}\right\},\left\{z_{\lambda}\right\}\right)$ ?

As an analogue of our arithmetic situation, let $\Lambda$ be the set of all free homotopy classes and $Y_{\lambda}$ a simple loop representing $\lambda$. In contrast to the arithmetic case, we cannot hope to recover the knot itself since the quandle is determined by the knot group and the knot group does not determine the knot. Also, if we replace the universal cover by the universal abelian cover, the resulting quandle seems to give little information since a homology class can contain countably many free homotopy classes.

\section{Automorphism groups}

For a topological quandle $Q$, let us denote by $\operatorname{Aut}(Q)$ the group of homeomorphic automorphisms of $Q$. Our aim in this section is to study $\operatorname{Aut}(Q)$ for topological quandles $Q$ associated to Galois covers.

Let $X$ be a normal, separated and integral scheme of finite type over $\mathbb{Z}, \mathcal{M}$ a set of closed points of $X$, and $\tilde{X}$ a Galois cover of $X$ unramified over $\mathcal{M}$, possibly of infinite degree. Denote $Q(\tilde{X} / X, \mathcal{M})$ by $Q$ and $\operatorname{Aut}(\tilde{X} / X)$ by $G$. Write $\pi: Q \rightarrow \mathcal{M}$ and $c: \mathcal{M} \rightarrow \operatorname{Spec} \mathbb{Z}$ for the natural maps.

If $G$ is abelian and $a$ is an element of $\mathcal{M}$, the Frobenius automorphism associated to $x \in \pi^{-1}(a)$ is independent of $x$, so let $s_{a}$ denote it.

\section{PROPOSITION 5.1.}

(1) If $G$ is abelian, a choice of a section of $\pi: Q \rightarrow \mathcal{M}$ determines an action of $\operatorname{Aut}(X, \mathcal{M})$ on $Q$.

(2) If $G$ is abelian, then there is a natural faithful action of $\prod_{a \in \mathcal{M}} G / \overline{\left\langle s_{a}\right\rangle}$ on $Q$.

(3) If $X=\operatorname{Spec} \mathcal{O}_{K} \backslash S$ for a number field $K$ and a finite set $S$ of primes and $\mathcal{M}$ has density one, then there is a natural homomorphism $\operatorname{Aut}(Q) \rightarrow S_{\mathcal{M}}$, where $S_{\mathcal{M}}$ is the (discrete) group of bijections from $\mathcal{M}$ to $\mathcal{M}$. 
If $G$ is abelian, then the kernel is isomorphic to $\prod_{a \in \mathcal{M}} G / \overline{\left\langle s_{a}\right\rangle}$ via the action in (2). In other words, there is a natural exact sequence

$$
1 \rightarrow \prod_{a \in \mathcal{M}} G / \overline{\left\langle s_{a}\right\rangle} \rightarrow \operatorname{Aut}(Q) \rightarrow S_{\mathcal{M}}
$$

Proof. Recall that $Q$ is isomorphic to $Q\left(G,\left\{\overline{\left\langle s_{x_{a}}\right\rangle}\right\}_{a \in \mathcal{M}},\left\{s_{x_{a}}\right\}_{a \in \mathcal{M}}\right)$ where $a \mapsto x_{a}$ is a section of $\pi$ (Proposition 3.8).

(1) If $G$ is abelian, the group $\operatorname{Aut}(X, \mathcal{M})$ acts on $G$ and $\mathcal{M}$ with the property $\sigma\left(s_{a}\right)=$ $s_{\sigma(a)}$ for $\sigma \in \operatorname{Aut}(X, \mathcal{M})$ and $a \in \mathcal{M}$. Thus the assertion follows from Lemma 5.2(1).

(2) This is immediate from Lemma 5.2(2).

(3) Apply Lemma 5.2(3) noting that $\operatorname{Inn}(Q)$ is dense in $\gamma(G)$ by Corollary 4.3 .

LEMMA 5.2. Let $G$ be a topological group, $\left\{z_{\lambda}\right\}_{\lambda \in \Lambda}$ a family of elements of $G$, and $\left\{H_{\lambda}\right\}_{\lambda \in \Lambda}$ a family of closed subgroups of $G$ such that $z_{\lambda} \in H_{\lambda}$ and $H_{\lambda}$ is contained in the centralizer of $z_{\lambda}$. Let $Q=Q\left(G,\left\{H_{\lambda}\right\},\left\{z_{\lambda}\right\}\right)$.

(1) If $G^{\prime}$ is a group acting on $G$ and $\Lambda$ with $\sigma\left(z_{\lambda}\right)=z_{\sigma(\lambda)}$ and $\sigma\left(H_{\lambda}\right)=H_{\sigma(\lambda)}$ for any $\sigma \in G^{\prime}$ and $\lambda \in \Lambda$, then $G^{\prime}$ acts on $Q$ by $\sigma\left(x H_{\lambda}\right):=\sigma(x) H_{\sigma(\lambda)}$.

(2) If $G$ is abelian, then there is a faithful action of $\prod_{\lambda \in \Lambda} G / H_{\lambda}$ on $Q$.

If $\left(Q^{\prime}, G\right)$ is an augmented quandle, $\Lambda=G \backslash Q^{\prime},\left\{q_{\lambda}\right\}_{\lambda \in \Lambda}$ is a complete system of representatives, $H_{\lambda}=\operatorname{Stab}_{G}\left(q_{\lambda}\right)$ and $z_{\lambda}=\varepsilon\left(q_{\lambda}\right)$, then the action of $\prod_{\lambda \in \Lambda} G / H_{\lambda}$ on $Q^{\prime}$ induced via the isomorphism of Proposition 2.9 does not depend on the choice of $q_{\lambda}$.

(3) Assume that $G$ is compact and write $\gamma: G \rightarrow C(Q, Q)$ for the map defined by the left translations. If $\operatorname{Inn}(Q)$ is dense in $\gamma(G)$, then there is a natural homomorphism $\operatorname{Aut}(Q) \rightarrow S_{\Lambda}$.

If $G$ is abelian, then the kernel is isomorphic to $\prod_{\lambda \in \Lambda} G / H_{\lambda}$ via the action in (2).

Proof. Recall that $Q=\bigsqcup_{\lambda \in \Lambda} G / H_{\lambda}$ (Example 2.4).

(1) Note that $\sigma(x) H_{\sigma(\lambda)}$ is equal to the set-theoretic image $\sigma\left(x H_{\lambda}\right)$ from the assumption that $\sigma\left(H_{\lambda}\right)=H_{\sigma(\lambda)}$. Thus the action is well defined.

We have

$$
\sigma\left(x H_{\lambda} \triangleright y H_{\mu}\right)=\sigma\left(x z_{\lambda} x^{-1} y H_{\mu}\right)=\sigma\left(x z_{\lambda} x^{-1} y\right) H_{\sigma(\mu)}
$$

and

$$
\sigma\left(x H_{\lambda}\right) \triangleright \sigma\left(y H_{\mu}\right)=\sigma(x) H_{\sigma(\lambda)} \triangleright \sigma(y) H_{\sigma(\mu)}=\sigma(x) z_{\sigma(\lambda)} \sigma(x)^{-1} \sigma(y) H_{\sigma(\mu)} .
$$

These are equal from the assumption $\sigma\left(z_{\lambda}\right)=z_{\sigma(\lambda)}$, so $G^{\prime}$ acts on $Q$ by quandle automorphisms.

(2) For $u=\left(u_{\lambda} H_{\lambda}\right) \in \prod_{\lambda \in \Lambda} G / H_{\lambda}$, we define $u \cdot x H_{\lambda}:=u_{\lambda} x H_{\lambda}$. Noting that $x H_{\lambda} \triangleright$ $y H_{\mu}=z_{\lambda} y H_{\mu}$, it is easy to see that $\prod_{\lambda \in \Lambda} G / H_{\lambda}$ acts on $Q$ by quandle automorphisms. This action is obviously faithful.

When $Q$ comes from an augmented quandle $Q^{\prime}$, first note that $\varepsilon\left(q_{\lambda}\right)$ and $\operatorname{Stab}_{G}\left(q_{\lambda}\right)$ are independent of the choice of $q_{\lambda}$. The isomorphism $\varphi_{q}: Q \stackrel{\sim}{\rightarrow} Q^{\prime}$ depends on the choice of $\left\{q_{\lambda}\right\}$, and if $\left\{q_{\lambda}^{\prime}\right\}$ is another system of representatives related with $\left\{q_{\lambda}\right\}$ by $q_{\lambda}^{\prime}=g_{\lambda} q_{\lambda}$, $g_{\lambda} \in G$, then $\varphi_{q}^{-1} \circ \varphi_{q^{\prime}}$ is equal to the translation by $\left(g_{\lambda} H_{\lambda}\right)$. This commutes with the action of $\prod_{\lambda \in \Lambda} G / H_{\lambda}$, so the actions on $Q^{\prime}$ induced by $\varphi_{q}$ and $\varphi_{q^{\prime}}$ are the same. 
(3) Note that $s_{x H_{\lambda}}$ is the left translation by $x z_{\lambda} x^{-1}$, so we always have $\operatorname{Inn}(Q) \subseteq \gamma(G)$.

Let $\varphi$ be an element of $\operatorname{Aut}(Q)$ and $q=x H_{\lambda}$ an element of $Q$. Then we have $\varphi(\operatorname{Inn}(Q) q) \subseteq \operatorname{Inn}(Q) \varphi(q)$. If $\operatorname{Inn}(Q)$ is dense in $\gamma(G)$, then we have $\varphi\left(G / H_{\lambda}\right)=\varphi(G q) \subseteq$ $G \varphi(q)$ by continuity. We see the other inclusion by considering $\varphi^{-1}$. Thus $\varphi$ maps $G / H_{\lambda}$ to some $G / H_{\mu}$, and induces a permutation on $\Lambda$.

The kernel of $\operatorname{Aut}(Q) \rightarrow S_{\Lambda}$ consists of automorphisms preserving each $G / H_{\lambda}$. Assume that $G$ is abelian and let $\varphi$ be such an automorphism. If $q \in G / H_{\lambda} \subseteq Q$, then $s_{q}$ is the left translation by $z_{\lambda}$. By assumption, $\varphi(q)$ is in the same $G / H_{\lambda}$, so we have $s_{q}=s_{\varphi(q)}$. From $\varphi \circ s_{q}=s_{\varphi(q)} \circ \varphi$ it follows that $\varphi$ commutes with $\operatorname{Inn}(Q)$. By continuity it also commutes with $G$. Let $u_{\lambda}$ be such that $\varphi\left(H_{\lambda}\right)=u_{\lambda} H_{\lambda} \in G / H_{\lambda}$ and write $u=\left(u_{\lambda} H_{\lambda}\right) \in \prod_{\lambda \in \Lambda} G / H_{\lambda}$. Then we have $\varphi\left(x H_{\lambda}\right)=x \varphi\left(H_{\lambda}\right)=x u_{\lambda} H_{\lambda}=u_{\lambda} x H_{\lambda}=u \cdot x H_{\lambda}$ for any $x \in G$ and $\lambda \in \Lambda$, so $\varphi$ coincides with the action of $u$.

COROLlary 5.3. Let $K$ be either $\mathbb{Q}$ or a quadratic field, $\mathfrak{p}$ a prime in $K, X=\operatorname{Spec} \mathcal{O}_{K} \backslash$ $\{\mathfrak{p}\}$ and $\mathcal{M} \subseteq X$ a set of primes of density one. If $G:=\pi_{1}^{\mathrm{ab}}(X)$ is infinite, there is a natural exact sequence

$$
1 \rightarrow \prod_{a \in \mathcal{M}} G / \overline{\left\langle s_{a}\right\rangle} \rightarrow \operatorname{Aut}\left(Q^{\mathrm{ab}}(X, \mathcal{M})\right) \rightarrow \prod_{l \in \operatorname{Spec} \mathbb{Z}} S_{c^{-1}(l)}
$$

Furthermore, $\operatorname{Aut}\left(Q^{\mathrm{ab}}(X, \mathcal{M})\right)$ has a subgroup isomorphic to $\operatorname{Aut}(X, \mathcal{M})$, and, except in the case where $K$ is real quadratic, we have

$$
\operatorname{Aut}\left(Q^{\mathrm{ab}}(X, \mathcal{M})\right)=\operatorname{Aut}(X, \mathcal{M}) \ltimes \prod_{a \in \mathcal{M}} G / \overline{\left\langle s_{a}\right\rangle} .
$$

Proof. By the main theorem, an automorphism $\varphi$ of $Q^{\mathrm{ab}}(X, \mathcal{M})$ commutes with $c \circ \pi$. In other words, the permutation on $\mathcal{M}$ induced by $\varphi$ preserves fibers of $c$, hence the first assertion.

By the previous proposition, a choice of section of $\pi$ gives a homomorphism $\operatorname{Aut}(X, \mathcal{M}) \rightarrow \operatorname{Aut}\left(Q^{\mathrm{ab}}(X, \mathcal{M})\right)$. The composite $\operatorname{Aut}(X, \mathcal{M}) \rightarrow S_{\mathcal{M}}$ corresponds to the natural action on $\mathcal{M}$, so it is injective. By the main theorem, $\operatorname{Aut}(Q)$ is generated by $\operatorname{Aut}(X, \mathcal{M})$ and automorphisms preserving fibers of $\pi$ except in the real quadratic case, hence the second assertion.

Example 5.4. If $K$ is quadratic, $\prod_{l \in \operatorname{Spec} \mathbb{Z}} S_{c^{-1}(l)}$ is much bigger than $\operatorname{Aut}(X, \mathcal{M})$. In the real quadratic case, it is possible that $\operatorname{Aut}\left(Q^{\mathrm{ab}}(X, \mathcal{M})\right) \rightarrow \prod_{l \in \operatorname{Spec} \mathbb{Z}} S_{c^{-1}(l)}$ is surjective.

In fact, let $K=\mathbb{Q}(\sqrt{5})$ and $\mathfrak{p}=3 \mathcal{O}_{K}$, which is a prime ideal, and $\mathcal{M}$ the set of primes of $K$ different from $\mathfrak{p}$ and $(\sqrt{5})$.

Let $\tilde{K}$ be the maximal abelian extension unramified outside $\mathfrak{p}$. It contains $K\left(\zeta_{3} \infty\right)$. We have $K \cap \mathbb{Q}\left(\zeta_{3} \infty\right)=\mathbb{Q}$ since $K$ is unramified over 3. Therefore $\operatorname{Gal}\left(K\left(\zeta_{3} \infty\right) / K\right) \cong$ $\operatorname{Gal}\left(\mathbb{Q}\left(\zeta_{3} \infty\right) / \mathbb{Q}\right) \cong \mathbb{Z}_{3}^{\times} \cong(\mathbb{Z} / 2 \mathbb{Z}) \times \mathbb{Z}_{3}$.

Since $K$ has narrow class number one, $\operatorname{Gal}(\tilde{K} / K)$ is isomorphic to $\mathcal{O}_{K_{\mathfrak{p}}}^{\times} / \overline{\mathcal{O}_{K}^{\times,+}}$. The group $\mathcal{O}_{K_{\mathfrak{p}}}^{\times}$can be decomposed as $\mu_{8} \times\left(1+3 \mathcal{O}_{K}\right)$. There is a totally positive unit $\gamma:=$ $\{(1+\sqrt{5}) / 2\}^{2}$, and it has order four modulo $1+3 \mathcal{O}_{K}$. We have $\gamma^{4}=1+3 \cdot(15+7 \sqrt{5}) / 2$, which spans a primitive $\mathbb{Z}_{3}$-submodule of $1+3 \mathcal{O}_{K}$. Thus $\mathcal{O}_{K_{\mathfrak{p}}}^{\times} / \overline{\mathcal{O}_{K}^{\times,+}}$is isomorphic to a quotient of $(\mathbb{Z} / 2 \mathbb{Z}) \times \mathbb{Z}_{3}$. It follows that the surjection $\operatorname{Gal}(\tilde{K} / K) \rightarrow \operatorname{Gal}\left(K\left(\zeta_{3^{\infty}}\right) / K\right)$ is in fact an isomorphism, i.e. $\tilde{K}=K\left(\zeta_{3} \infty\right)$. 
Let $l$ be a prime that splits as $\mathfrak{l} \cdot \overline{\mathfrak{l}}$ in $K$. Then $\mathfrak{l}$ and $\overline{\mathfrak{l}}$ define the same element of $G:=\operatorname{Gal}(\tilde{K} / K)$, i.e. the automorphism given by $\zeta_{3^{n}} \mapsto \zeta_{3^{n}}^{l}$. Thus we can exchange the fibers of $Q^{\mathrm{ab}}(X, \mathcal{M})$ over $\mathfrak{l}$ and $\overline{\mathfrak{l}}$ by a quandle automorphism. In this case we have $\operatorname{Aut}\left(Q^{\mathrm{ab}}(X, \mathcal{M})\right)=\prod_{l \in \operatorname{Spec} \mathbb{Z}}\left(S_{c^{-1}(l)} \ltimes \prod_{\mathfrak{l} \in c^{-1}(l)} \mathbb{Z}_{3}^{\times} / \overline{\langle N(\mathfrak{l})\rangle}\right)$.

Acknowledgements. I would like to thank Makoto Matsumoto, Akio Tamagawa, and the referee for a previous version of this paper for their valuable comments from the point of view of anabelian geometry. I would also like to thank the referee for helpful comments concerning the relation between knot quandles and the current work.

This work was supported by JSPS KAKENHI Grant Number JP17K05204.

\section{REFERENCES}

[I14] A. Ivanov. On some anabelian properties of arithmetic curves. Manuscripta Math. 144(3) (2014), $545-564$.

[I15] A. Ishii. A multiple conjugation quandle and handlebody-knots. Topology Appl. 196 (2015), 492-500.

[J82] D. Joyce. A classifying invariant of knots, the knot quandle. J. Pure Appl. Algebra 23(1) (1982), $37-65$.

[M82] S. V. Matveev. Distributive groupoids in knot theory (in Russian). Mat. Sb. 119 (1982), 78-88 (English transl. Math. USSR-Sb. 47(1) (1984), 73-83).

[M08] S. Mochizuki. The geometry of Frobenioids. I. The general theory. Kyushu J. Math. 62(2) (2008), 293-400.

[M12] M. Morishita. Knots and Primes (Universitext). Springer, London, 2012.

[NSW08] J. Neukirch, A. Schmidt and K. Wingberg. Cohomology of Number Fields, 2nd edn (Grundlehren der Mathematischen Wissenschaften, 323). Springer, Berlin, 2008.

[R07] R. L. Rubinsztein. Topological quandles and invariants of links. J. Knot Theory Ramifications 16(6) (2007), 789-808.

[S66] J.-P. Serre. Dépendance d'exponentielles $p$-adiques. Séminaire Delange-Pisot-Poitou (Théorie des Nombres), 7è année, 1965/66, no. 15.

[T97] A. Tamagawa. The Grothendieck conjecture for affine curves. Compositio Math. 109(2) (1997), 135-194.

[W81] M. Waldschmidt. Transcendance et exponentielles en plusieurs variables. Invent. Math. 63(1) (1981), 97-127.

Nobuyoshi Takahashi

Department of Mathematics

Graduate School of Science

Hiroshima University

1-3-1 Kagamiyama

Higashi-Hiroshima 739-8526

Japan

(E-mail: tkhsnbys@hiroshima-u.ac.jp) 